\title{
Physics of nearshore bed pattern formation under regular or random waves
}

\author{
Sonja M. van Leeuwen, ${ }^{1,2}$ Nicholas Dodd, ${ }^{1}$ Daniel Calvete, ${ }^{3}$ and Albert Falqués ${ }^{3}$ \\ Received 28 June 2005; revised 9 November 2005; accepted 14 December 2005; published 28 March 2006.
}

[1] We present an investigation into the growth of nearshore, rhythmic patterns. A comprehensive linear stability model of the surf and shoaling zones is used to examine which type of pattern, transverse or crescentic bar, is likely to form under different wave conditions. In contrast to earlier studies we examine normal and near-normal incidence on a plane beach. In doing so we reproduce results of earlier, more restricted studies and thereby identify the physical mechanisms leading to the growth of different patterns. This paper also focuses on the role of random wave height distribution compared with regular waves and identifies conditions likely to lead to pattern growth. To this end, an amended wave height dissipation function is presented, which allows us to move between random and regular regimes. It is found that a sharply defined surf breakpoint leads to larger growth rates and crescentic-bar-type features. In contrast, a large spread in breaking gives rise to transverse bar patterns with reduced growth rates. Transverse bar alongshore spacing is typically about $1 / 4$ to $1 / 2$ the width of the surf zone, while crescentic bar spacing is larger, up to twice this width. It is also shown that pattern types are influenced by the wave height to depth ratio in the surf zone. This indicates that sites with substantial inner surf zone wave energy and thus greater energy available to move sediment will give rise to transverse bar patterns. A new, propagating mode is identified in such cases, which exists for normal wave incidence. Finally, the role of wave shoaling and wave refraction, either on the bed or on the currents is examined. Crescentic bars seem to be a very robust feature as they stem from the model even if those three effects are ignored. Thus the only essential feedback for their formation is the coupling between depthcontrolled breaking and the evolving bathymetry. In contrast, transverse bar formation is very sensitive to wave refraction being enhanced by refraction over the bed and weakened by refraction over the current.

Citation: van Leeuwen, S. M., N. Dodd, D. Calvete, and A. Falqués (2006), Physics of nearshore bed pattern formation under regular or random waves, J. Geophys. Res., 111, F01023, doi:10.1029/2005JF000360.

\section{Introduction}

[2] Rhythmic bed patterns can be found around the world in coastal areas. In the nearshore zone these patterns include beach cusps [Coco et al., 1999], mega cusps [Short, 1999], sand waves [Falqués and Calvete, 2005], transverse bars [Konicki and Holman, 2000], oblique bars [Ribas et al., 2003], and crescentic bars [Ruessink et al., 2000; van Enckevort et al., 2004]).

[3] Previous studies have described some fundamental mechanisms for bed pattern generation in the surf zone. Falqués et al. [1996] discern two different feedback pro-

\footnotetext{
${ }^{1}$ School of Civil Engineering, University of Nottingham, Nottingham, UK.

${ }^{2}$ Now at the Centre for Environment, Fisheries and Aquaculture Science, Lowestoft, UK.

${ }^{3}$ Departimento de Física Aplicada, Universitat Politécnica de Catalunya, Barcelona, Spain.

Copyright 2006 by the American Geophysical Union. 0148-0227/06/2005JF000360\$09.00
}

cesses of the bed perturbation on the hydrodynamics: the bed-surf mechanism (influence of the emerging bed on the incoming wavefield) and the bed-flow mechanism (effect of the emerging bed on the alongshore current). Falqués et al. [2000] applied a simple linear stability model to study the bed-surf mechanism on a plane, erodible beach with regular wave forcing. Focusing on the feedback of the emerging bed on the cross-shore point of wave breaking they found crescentic bar patterns. This research was extended by Caballeria et al. [2002] using a nonlinear finite difference model, who examined wave refraction over the bed pattern, showing that transverse bars can form. Crescentic bars were also found and analysis showed that both patterns are limits of the same instability mechanism. When hydromorphological or morphological diffusion is strong enough the transverse bars are damped (due to the short wavelengths) and crescentic bars emerge. Intermediate patterns showing both bar types were also found. Ribas et al. [2003] considered oblique incident waves on a plane beach. Not accounting for wave refraction they found that near-normal wave incidence can result in two different crescentic bar patterns, which 


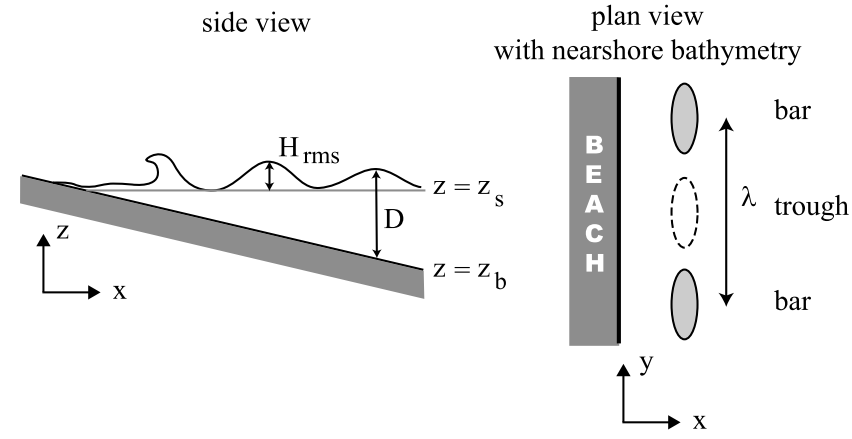

Figure 1. Model geometry and some variables.

form a complex conjugate solution (two patterns with equal temporal evolution but opposite directions of migration and obliquity compared to the breaker line position) in the limit of normal incidence.

[4] These studies identify important feedback mechanisms for rhythmic nearshore pattern formation. However, they are restricted: depth-limited waves (ie, wave height a constant proportion of the local depth) and regular wave forcing are assumed. Wave shoaling (over depth and current) and wave refraction on a current are not considered, with feedback onto the wavefield being solely via moving breakpoint and differential breaking due to changing water depth for Falqués et al. [2000] and Ribas et al. [2003]. Further, sediment availability due to wave stirring was imposed as a prescribed function, depending only on cross-shore location. In fact [see Falqués et al., 2000], the influence of the imposed stirring functions can be severe, due to the critical role played by depth-averaged or potential stirring (ratio of sediment stirring over depth). Caballeria et al. [2002] even reported contradictory results for an imposed stirring function and one based on the Bailard sediment transport formulation. Falqués et al. [2000] and Ribas et al. [2003] considered a constant sediment stirring function and an exponential one (increasing up to the point of wave breaking and decreasing beyond). However, these imposed stirring functions are not consistent with the hydrodynamics describing regular, depth-limited waves. Furthermore, the use of depth-limited, regular waves is unrealistic, as field conditions will be random. Calvete et al. [2005] have since used the Thornton and Guza [1983] model of dissipation in a comprehensive linear stability model, to examine growth of bed forms on a barred beach. It remains unclear, however, what the effects are of (1) the spreading out of the breakpoint into a finite region and (2) the relaxation of the depth-limited wave condition.

[5] This paper presents an investigation into these issues. However, the examination of a barred beach obscures these phenomena, as breaking is either concentrated at the offshore bar face or (smaller) waves penetrate to the very inner surf zone, so that these effects are difficult to disentangle and control. Therefore we apply the linear stability model of Calvete et al. [2005] to a plane beach and control dissipation by use of a modified dissipation formula, first used by van Leeuwen et al. [2004] to make Monte Carlo simulations of wave height transformation, and presented here for the first time in a full journal paper.

[6] In section 2 we describe the model used here, with focus on the dissipation function. Next, four different wave types are introduced, representing a smooth transition from depth-limited regular waves to fully random waves. We present results for depth-limited regular waves in section 4, in which the aforementioned modified dissipation is used first to reproduce earlier results, and then to isolate physical effects, thereby illustrating mechanisms for bed form growth. In section 5 we relax our assumption of regular waves and examine the bed forms that emerge for more random wave types. We present a discussion in section 6, and our conclusions in section 7 .

\section{Model Description}

[7] The study was carried out using the linear stability model MORFO60. This model is based on the shallow water equations [see Calvete et al., 2005, for details]. We consider a plane beach (default slope 0.02), which extends up to $4 \mathrm{~km}$ offshore, after which the profile becomes flat (constant depth $80 \mathrm{~m}$ ). The origin is chosen at the intersection of the mean water level (without setup) with the bed (see Figure 1).

[8] The cross-shore and alongshore coordinates are given by $\vec{x}=\left(x_{1}, x_{2}\right)=(x, y)$, while velocity is denoted by $\vec{v}=\left(v_{1}, v_{2}\right)$. Total depth is $D=z_{s}-z_{b}$ with $z_{s}$ the free surface and $z_{b}=-\beta x$ the planar bed. The governing equations are

$$
\begin{gathered}
\frac{\partial D}{\partial t}+\frac{\partial D v_{i}}{\partial x_{i}}=0 \\
\frac{\partial v_{i}}{\partial t}+v_{j} \frac{\partial v_{i}}{\partial x_{j}}=-g \frac{\partial z_{s}}{\partial x_{i}}-\frac{1}{\rho D} \frac{\partial}{\partial x_{j}}\left(S_{i j}^{\prime}-S_{i j}^{\prime \prime}\right)-\frac{\tau_{b i}}{\rho D}, \\
\frac{\partial E}{\partial t}+\frac{\partial}{\partial x_{i}}\left(\left(v_{i}+c_{g i}\right) E\right)=-S_{i j}^{\prime} \frac{\partial v_{j}}{\partial x_{i}}-\mathcal{D} \\
\frac{\partial \Phi}{\partial t}+\sigma+v_{i} \frac{\partial \Phi}{\partial x_{i}}=0 \\
\frac{\partial z_{b}}{\partial t}=-\frac{1}{1-p} \frac{\partial q_{i}}{\partial x_{i}}
\end{gathered}
$$

and comprise the equations of mass conservation, momentum (in the horizontal directions), energy density $E=\frac{1}{2} \rho g H^{2}$, wave phase $\Phi$ and sediment conservation. The equations are depthintegrated and averaged over a wave period. Repeated indices indicate summation with $i, j=1,2$ the two horizontal directions and $t$ is time. Here $S_{i j}^{\prime}$ is the wave radiation stress, $S_{i j}^{\prime \prime}$ the turbulent Reynolds stress, $\sigma$ the intrinsic frequency, $\vec{c}_{g}$ the group velocity and $\vec{\tau}_{b}$ the bed shear stress. The sediment flux vector is denoted $\vec{q}$ and dissipation due to wave breaking by $\mathcal{D}$. The bed porosity is $p=0.4$, the seawater density $\rho=1024 \mathrm{~kg} \mathrm{~m}^{-3}$ and $H$ the wave height in meters. Linear bottom friction is applied with

$$
\vec{\tau}_{b}=\left(\frac{2}{\pi}\right) \rho c_{d} u_{w o v} \vec{v}, \quad c_{d}=\left(\frac{0.4}{\ln \left(D / z_{r l}\right)-1}\right)^{2},
$$


where $z_{r l}=0.01 \mathrm{~m}$ is the bed roughness length, and $u_{w o v}$ the wave orbital velocity.

\subsection{Dissipation}

[9] The dissipation in a single wave $\left(\mathcal{D}_{1}\right)$ due to breaking is based on dissipation in a bore [Battjes and Janssen, 1978] and given by

$$
\mathcal{D}_{1}=\frac{\rho g \sigma B^{3} H^{3}}{8 \pi D}
$$

where $B$ determines the type of breaking (here $B=1.0$, a spilling breaker). The averaged dissipation $\overline{\mathcal{D}}$ for a random set of waves can then be determined by

$$
\overline{\mathcal{D}}=\int_{0}^{\infty} \mathcal{D}_{1} W P_{\text {Rayleigh }} d H .
$$

Here $P_{\text {Rayleigh }}=2 H / H_{r m s}^{2} e^{-\left(H / H_{r m s}^{2}\right)}$ is the probability density function of the Rayleigh distribution [see Thornton and Guza, 1983], and $W$ is a weighting function that describes the proportion of waves that have already broken for a given depth. Thornton and Guza [1983] used data from Torrey Pines Beach, California to derive their breaker index $\gamma_{b}=$ 0.42 and weighting function, resulting in

$$
W=\left(\frac{H_{r m s}}{\gamma_{b} D}\right)^{m}\left(1-e^{-\left(\frac{H}{\gamma_{b} D}\right)^{2}}\right)
$$

The frequencies of individual waves are assumed to be narrow banded with peak frequency $f_{p}$. Comparison with data gave a best fit for $m=2$. The power 2 in the second part of (9) was chosen to allow analytical integration of (8). The breaker index value $(0.42)$ is typical of such values measured in the field, and contrasts markedly with values obtained for regular waves $(0.7-1.0$ [see $\mathrm{Mei}, 1989])$. This is primarily due to the mixture of broken and nonbroken waves that occur in field observations. As a result, the wave height transformation predicted by the Thornton and Guza [1983] model is not depth-limited. Note that the expression is forced by $H_{r m s}$, the root-mean-square wave height of the imposed Rayleigh distribution.

[10] Roelvink [1993] used a similar approach but allowed for numerical integration of (8) over all the wave heights. His weighting function is

$$
W=\left(1-e^{-\left(\frac{H}{\gamma_{b} D}\right)^{n}}\right)
$$

which assumes that no information about the whole wave train $\left(H_{r m s}\right)$ is known to each individual wave, thus allowing breaking to occur at one point for high values of $n$. Roelvink [1993] calibrated $n$ using mainly laboratory data, characterized by $0.52<\gamma_{b}<0.66$. The comparison showed little sensitivity to $n$, with equal accuracy obtained for $n=10$ and 20.

[11] Here, to describe breaking of regular waves we use

$$
W=\left(\frac{H}{\gamma_{b} D}\right)^{m}\left(1-e^{-\left(\frac{H}{\gamma_{b} D}\right)^{n}}\right)
$$

$$
\begin{aligned}
\mathcal{D} & =\mathcal{D}_{1} W \\
& =\frac{\rho g \sigma B^{3} H^{3}}{8 \pi D}\left(\frac{H}{\gamma_{b} D}\right)^{m}\left(1-e^{-\left(\frac{H}{\gamma_{b} D}\right)^{n}}\right),
\end{aligned}
$$

which is based on the Roelvink [1993] formula but complemented with the first part of the weighting function of Thornton and Guza [1983]: without data comparison the parameter $m$ is free. As such it can be used to describe different wave transformations. For large values of $n$ breaking will occur in one point, with decreasing values of $n$ representing a smooth transition to a more random wave regime. Note that the expression is not integrated and thus depends on the individual wave height $H$. The parameter $m$ controls the ratio $H / D$ after breaking, with a large value of $m$ resulting in a depth-limited wave. Thus the choice $m, n \gg 1$ mimics depth-limited waves without directly prescribing $H$ as a function of depth within this region. Decreasing values of $m$ allows for increase of the wave height over depth ratio in the surf zone. The choice $m=0, n \gg 1$ describes the dissipation due to one wave as based on the bore dissipation model continuously throughout the domain.

[12] Finally, the break point is denoted $x_{b}$. For random waves $x_{b}$ is defined here as the location of maximum dissipation. Because of the mild slope (1:50) and wave period (6s) only saturated conditions are found for all wave types, i.e., energy within the surf zone does not increase with increasing offshore energy. For a parametric dissipation expression allowing short wave energy to reach the shoreline, see Baldock et al. [1998].

\subsection{Sediment Transport}

[13] We use the sediment transport formula of SoulsbyVan Rijn, which is a total load formula containing a threshold of motion:

$$
\begin{array}{cl}
\vec{q}=A_{s}\left[\left(|\vec{u}|^{2}+\frac{0.018}{c_{d}} u_{\text {wov }}^{2}\right)^{0.5}-u_{\text {crit }}\right]^{2.4}\left(\vec{u}-\gamma u_{\text {wov }} \overrightarrow{\nabla h}\right) \\
\quad \operatorname{if}\left(|\vec{u}|^{2}+\frac{0.018}{c_{d}} u_{\text {wov }}^{2}\right)>u_{\text {crit }} \\
=0 \quad \text { otherwise }
\end{array}
$$

with $u_{\text {crit }}$ the critical velocity for sediment motion (depending on median grain size), $\gamma=1.0$ a diffusion coefficient and $A_{s}$ a constant depending on the median grain size and the relative density of sediment [Soulsby, 1997].

[14] The sediment flux $\vec{q}$ is rewritten as

$$
\vec{q}=\alpha(x) \vec{u}-\gamma(x) \vec{\nabla} h
$$

where $\alpha(x)$ is the wave stirring function (sediment brought into suspension by waves and currents and transported by the currents) and $\gamma(x)$ is the morphological diffusion. This diffusion is only applied to the bed disturbance $h$; we assume that offshore transport of sediment due to beach slope and undertow is compensated by onshore transport due to wave asymmetry and infragravity motions. 
[15] Expression (14) is also identical to that of Falqués et al. [2000] and similar studies, and so allows us to make direct comparisons. Substituting equation (13) in equation (14) provides the expressions for wave stirring function $\alpha(x)$ and morphological diffusion function $\gamma(x)$ :

$$
\begin{gathered}
\alpha(x)=A_{s}\left[\left(|\vec{u}|^{2}+\frac{0.018}{c_{d}} u_{\text {wov }}^{2}\right)^{0.5}-u_{\text {crit }}\right]^{2.4} \\
\gamma(x)=\alpha(x) \gamma u_{\text {wov }}
\end{gathered}
$$

It can be shown from (14) and (5) [see Falqués et al., 2000] that bed perturbation growth is linked to the depth-averaged (or potential) wave stirring function $\alpha / D$ : a perturbation will grow if the gradient in the depth-averaged wave stirring function opposes the flow direction over the positive bed perturbation. We will refer extensively to depth-averaged stirring later.

\subsection{Linear Stability Analysis}

[16] In linear stability analysis the variables are split into an equilibrium part (basic state, e.g., $D_{e q}$ ) plus a perturbation (e.g., $d$ ). The equilibrium state problem is solved by integration inshore [Calvete et al., 2005]. The shoreline position depends on the setup and is determined by the condition $D \leq 10 \mathrm{~cm}$ to avoid convergence problems. The perturbations are assumed to be periodic in the alongshore direction and possess a complex exponential behavior in time, thus leaving their cross-shore profile to be determined (e.g., $d^{\prime}(x)$ ). They are also assumed to be small compared to the equilibrium variables:

$$
\begin{gathered}
D(x, y, t)=D_{e q}(x)+d(x, y, t), \quad \text { with } d(x, y, t) \ll D_{e q}(x) \\
d(x, y, t)=d^{\prime}(x) e^{i \kappa y+\Omega t} .
\end{gathered}
$$

Similar expressions result for the other unknown variables $\left(E, \vec{v}, z_{b}, \Phi\right)$. The linear stability problem thus yields an eigenvalue problem which is solved by a spectral method using rational Chebychev expansion. Here $\Omega=\Omega_{r}+i \Omega_{i}$ is the complex eigenvalue of the system, with the real part denoting growth rate and the imaginary part indicating alongshore migration of the pattern. The cross-shore perturbation profiles are the associated eigenfunctions. The alongshore wave number $\kappa$ is a model input parameter: for each given $\kappa$ the eigenvalue problem is solved and an eigenvalue is obtained together with the associated cross-shore profiles. One such solution is called a mode of the system. The mode with the largest growth rate will be dominant over the other modes and is thus expected to appear in nature. The exclusion of diffraction effects leads to an approximated, natural limit of the possible alongshore perturbation wavelengths: $\lambda \sim 2 \pi / \kappa \leq \lambda_{\text {waves }}$ with $\lambda_{\text {waves }}$ the wavelength of the surface gravity waves. The use of linearized equations implies that perturbation amplitudes will be unknown: resulting perturbations are scaled to represent a maximum bed perturbation of $\pm 0.5 \mathrm{~m}$. Note that the condition $d(x, y, t) \ll D_{e q}(x)$ limits the applicability of the results to initial formation of the features.

[17] Basic state boundary conditions include prescribed values of the offshore wave height, period and incidence angle. Default values are $H_{r m s, \infty}=H_{\infty}=1.0 \mathrm{~m}, T_{p, \infty}=$ $T_{\infty}=6 \mathrm{~s}$ and $\theta_{\infty}=0^{\circ}$. The equilibrium velocities and free surface elevation far offshore are assumed zero, with $v_{2}=0$ at the shoreline. The perturbations are all assumed to be zero at the offshore boundaries while $\vec{v}^{\prime}, h^{\prime}=0$ at the shoreline (second-order equations).

\section{Different Wave Types}

[18] Here we define the four types of waves applied in this paper: (1) depth-limited regular waves, (2) regular waves, (3) intermediate waves, and (4) random waves.

[19] For depth-limited regular waves we use (12) with $n=50, m=30, \gamma_{b}=0.8$. The choice of $n, m$ is based on the desired dissipation profile (depth-limited waves, breaking at one point with $\gamma_{b}$ from Horikawa [1988]), taking into consideration numerical limitations caused by sharp cross-shore gradients. We also use (12) for regular waves but with $n=50, m=0, \gamma_{b}=0.8$, thus ensuring breaking at one point while surf zone wave height is no longer depth restricted. As such it follows the classical description of regular waves [Battjes and Janssen, 1978]. For intermediate waves we relax conditions regarding breaking at one point by taking (12) with $n=10, m=$ 0 and $\gamma_{b}=0.55$, the latter value being for individual waves based on laboratory data of random waves. Thus these waves provide a link between regular and random waves. Finally, random wave dissipation is from the Thornton and Guza [1983] model (equation (9) with $\gamma_{b}=0.42$ ). Cross-shore dissipation profiles and wave height for the different wave types are shown in Figure 2 for the equilibrium state. This equilibrium is the cross-shore balance between pressure gradient due to wave setup and radiation stress. Regular waves break at one point, $x_{b} \sim 80 \mathrm{~m}$. Wave shoaling causes a wave height increase of $\sim 20 \%$ before breaking, accompanied by setdown. More random wave types are characterized by decreased shoaling and setup, with the fully random waves showing a complete lack of shoaling due to strong dissipation offshore. The result clearly shows the spatial spread in random wave dissipation. The depthaveraged stirring shows a strong decrease in both magnitude and slope when going from depth-limited regular waves to random ones. The difference between depth-limited regular waves and regular waves is minimal, with only a small increase of $H / D$ for the innermost surf zone. The wave conditions lead to a natural depth limitation of wave height within the surf zone, due to the mild slope and wave period [Baldock et al., 1998; van Leeuwen et al., 2004].

[20] In Figure 3 we also present the profiles for different wave types using the same breaker index, $\gamma_{b}=0.8$. Clearly, more random waves have a decreased setup, due to the spread in dissipation, causing an offshore shift in the location where wave height first starts to decay, thus increasing surf zone width. The decrease in setup leads to a decrease in depth at the same point and, with equal wave heights, an increase in $H / D$. This occurs for intermediate waves. For fully random waves the surf zone wave height is decreased 


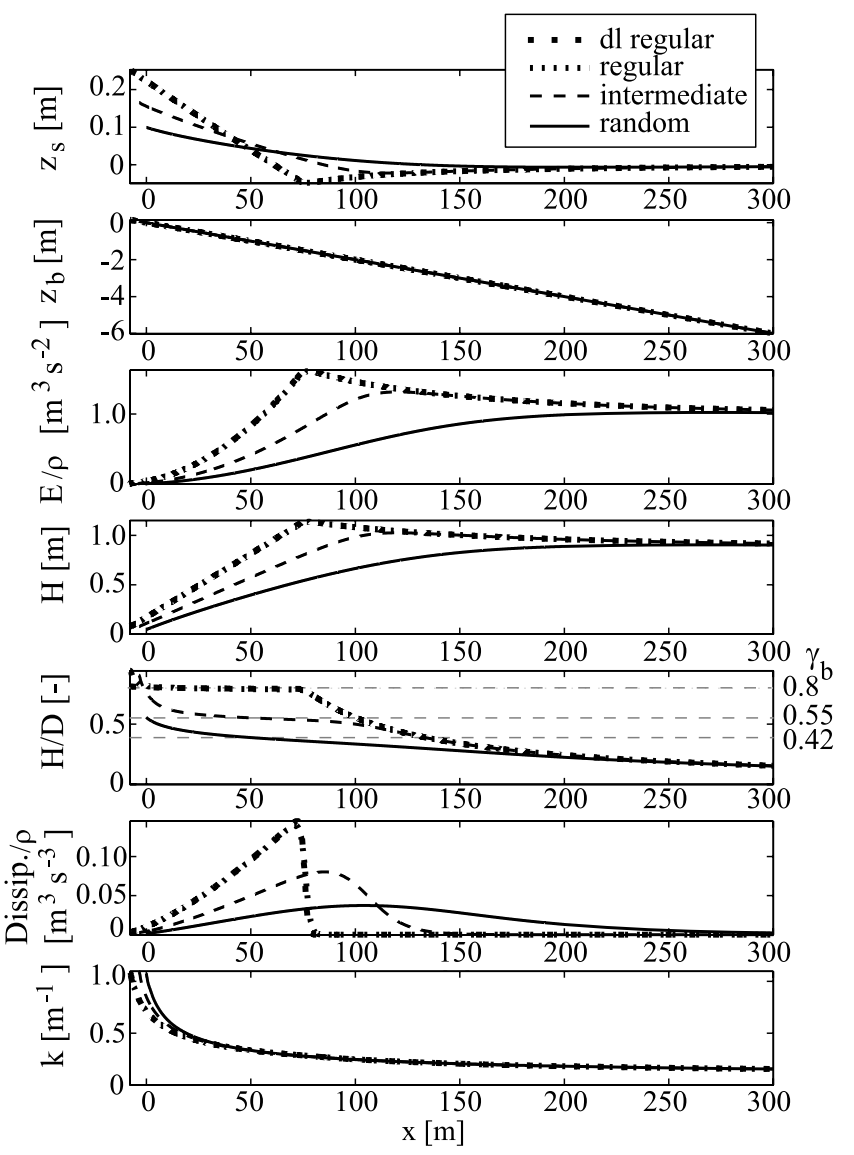

(a)

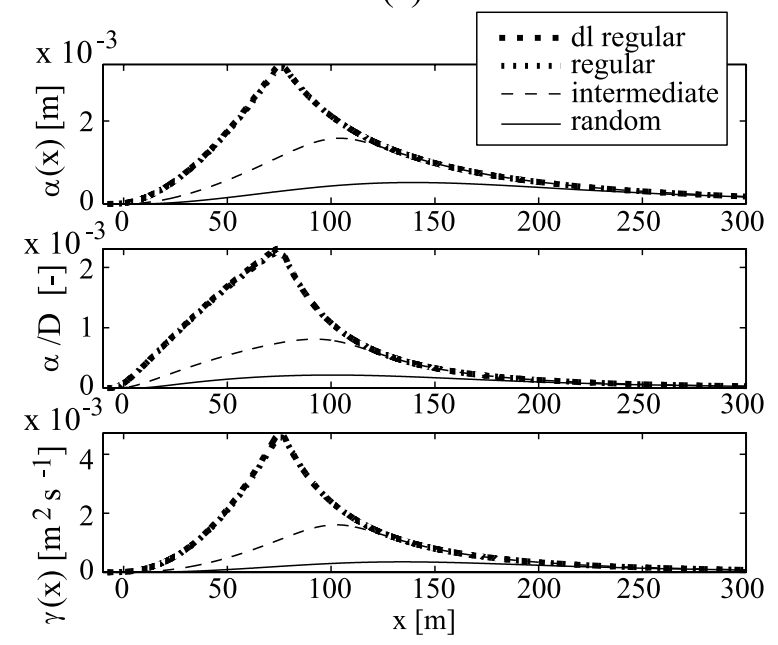

(b)

Figure 2. Cross-shore equilibrium profiles for different types of waves, with varying breaker indices $\left(H_{\infty}=1.0 \mathrm{~m}\right.$, $T_{\infty}=6 \mathrm{~s}$, and $\theta_{\infty}=0^{\circ}$ ): (a) hydrodynamical variables and (b) morphodynamical variables. Note that the position of the origin is kept fixed, as different wave types lead to different setup heights and thus inshore penetration. due to strong offshore dissipation, leading to a much smaller increase of $H / D$ in the surf zone, even though the setup is further reduced.

\section{Bed Pattern Generation due to Depth-Limited Regular Waves}

[21] We begin by considering depth-limited waves. For default parameters see section 2 . To reproduce the results of Falqués et al. [2000] and Caballeria et al. [2002], the model is initially reduced to represent simpler hydrodynamics. We focus on wave refraction over the emerging bed and current, as it is expected that these feedbacks play an important part in pattern generation [Caballeria et al., 2002]. The studies mentioned above exclude or only partially include these effects; wave shoaling was not accounted for, and will also be examined.

[22] The governing equations are given by equations (1)(5) in section 2. Shoaling is excluded by eliminating all feedback of the perturbations on to the wave energy equation except through the dissipation (energy and depth). Equation analysis shows that this is also the governing balance for the full equations. Refraction over the current is eliminated by excluding feedback of the perturbed current on to the phase equation. We exclude depth perturbation terms in the phase equation, thus eliminating refraction over depth.

[23] Thus we arrive at a model that, in the main balances, should be close to that used by Falqués et al. [2000]. Different feedback mechanisms are then added one by one to examine their influence on the system and predicted bed pattern. Note that the changes only apply to the feedback mechanisms through perturbations: for example, when wave
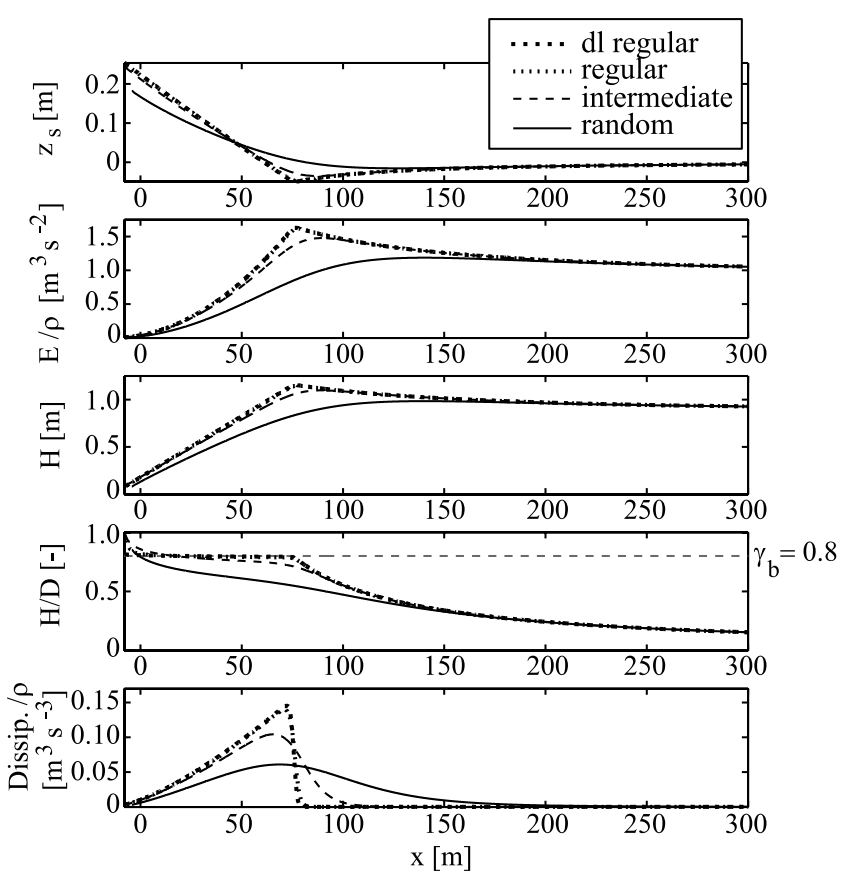

Figure 3. Cross-shore equilibrium profiles for different types of waves using the same breaker index of $\gamma_{b}=0.8$. Again, the position of the origin is kept fixed. 


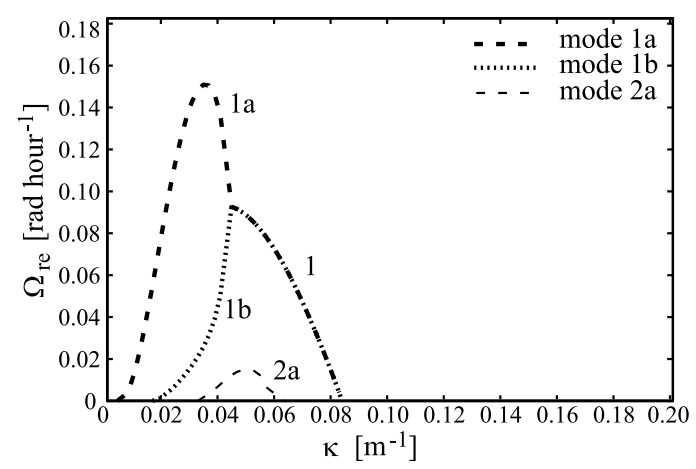

(a)

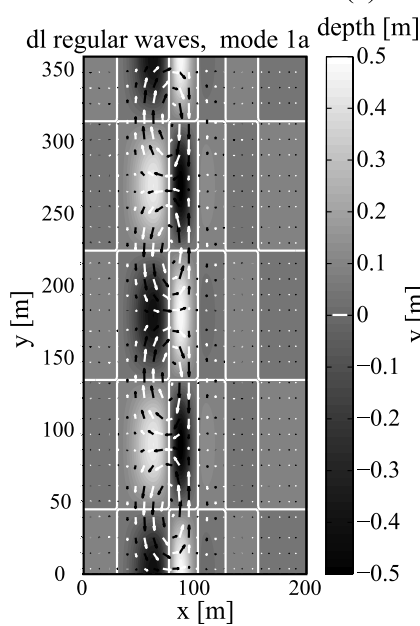

(b)

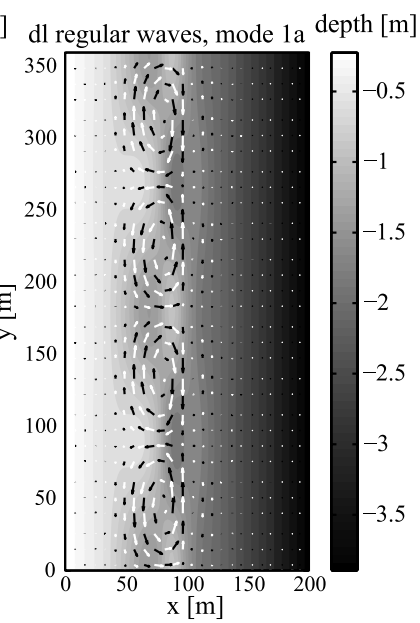

(c)
Figure 4. Results for depth-limited regular waves $(n=50$, $m=30)\left(H_{\infty}=1.0 \mathrm{~m}, T_{\infty}=6 \mathrm{~s}\right.$, and $\left.\theta_{\infty}=0^{\circ}\right)$ excluding refraction and shoaling of the perturbations: (a) growth rate curve and (b and c) plan view of the fastest growing bed pattern $(\kappa \sim 0.035 \mathrm{rad} / \mathrm{m})(\mathrm{m}$; shoals are light and troughs are dark) and velocity field (arrows) for the perturbations only (Figure $4 b$ ) and the total profile (Figure 4c, equilibrium plus perturbations). The white line marks the zero perturbation line.

shoaling is neglected, the equilibrium profile still experiences a wave height increase due to shoaling.

[24] Last, note that the friction coefficient is depthdependent here; the earlier studies applied a constant drag coefficient $\left(c_{d}=0.01\right.$ for Falqués et al. [2000] and Ribas et al. [2003] and $c_{d}=0.002$ for Caballeria et al. [2002]). Numerical experiments show that using a constant drag coefficient in the present model causes the depth-averaged stirring to decrease in the offshore direction. A depthdependent drag coefficient [see Calvete et al., 2005] allows depth-averaged stirring to increase up to $x=x_{b}$ and decrease thereafter, consistent with short wave induced stirring. A roughness length of $1 \mathrm{~cm}$ results in $c_{d} \sim 0.01$ at $x=x_{b}$ for default parameter values. As such $r=c_{d} / \beta=0.5$ is in line with the studies of Falqués et al. [2000] and Ribas et al. [2003] at this position.

[25] The basic state profiles remain unchanged for all results for regular, depth-limited waves (see Figure 2). Cross-shore equilibrium profiles for $\alpha, \alpha / D$ and $\gamma$ (see Figure 2) are characterized by a clear maximum at $x=x_{b}$.
The $\alpha(x)$ profile shows that the imposed sediment stirring in previous studies (exponential up to the breaker line and decreasing beyond) is quite realistic. Note that this stirring profile is characteristic for depth-limited waves.

\subsection{Excluding Wave Refraction and Shoaling}

[26] Here we exclude shoaling and wave refraction, as outlined above. Figure 4 shows the results of the linear stability analysis. The bed pattern resembles the crescentic bars of Falqués et al. [2000], with maximum bed perturbation located offshore of $x_{b}$ and alongshore wavelength $\lambda=$ $2 \pi / \kappa_{\max } \sim 2 x_{b}$. The e-folding timescale is $1 / \Omega_{r e} \sim 6.6$ hours, which is in line with that of Falqués et al. [2000] (1.8--14.0 hours for a steep beach; $\beta=0.05$, based on equation analysis). The growth rate curve shows three modes (1a, 1b, 2), two of which form a complex conjugate solution (1a and $1 \mathrm{~b}$ form mode 1 , hereafter called a double mode) for a small range of alongshore wave numbers $\left(\sim 0.04 \mathrm{rad} \mathrm{m}^{-1} \leq \kappa \leq 0.08 \mathrm{rad} \mathrm{m}^{-1}\right)$. These solutions have equal growth and migration rate magnitudes, but migrate in opposite directions. We return to these double modes later.

[27] Circulation cells occur with onshore flow over the shoals in the surf zone, as predicted by the instability mechanism [Falqués et al., 2000]. Near the shoreline very weak secondary circulation cells are observed [see Calvete et al., 2005]. Thus the model is able to reproduce earlier results.

\subsection{Influence of Wave Shoaling}

[28] Next, wave shoaling only is reincorporated. The resulting dominant bed pattern (results not shown) is characterized by elongated, crescentic bars located primarily in the shoaling zone. The large offshore extent of these features is due to wave shoaling: a positive bed perturbation in the shoaling zone causes a local increase in wave height compared to conditions further offshore. The increase in wave energy causes a local increase in cross-shore radiation stress, leading to a negative gradient in $S_{x x}^{\prime}$ in the offshore direction. The cross-shore momentum input over the shoal is $\sim-\frac{1}{\rho D} \frac{\partial S_{x x}^{\prime}}{\partial x}>0$, thus a positive force is acting on the water column which results in the generation of an offshore directed current. As depth-averaged stirring decreases in the shoaling zone the offshore current over the shoal leads to growth of the feature. The pattern extends from the breaker line to $\sim 500 \mathrm{~m}$ offshore (depth $\sim 10 \mathrm{~m}$ ) where shoaling first occurs. The e-folding timescale is now $\sim 6$ days. This substantial decrease in growth rate may be due to the movement of the instability into the shoaling zone, where gradients in depth-averaged stirring are smaller and therefore the instability mechanism less efficient.

\subsection{Influence of Wave Refraction Over Depth}

[29] The work by Niederoda and Tanner [1970] and Caballeria et al. [2002] shows that wave refraction over depth is an essential mechanism for transverse bar growth. Waves are focused over the shoal, where energy is then dissipated. The ensuing onshore flow over the entire shoal causes growth (if the stirring function is increasing in the offshore direction in the surf zone) of elongated patterns, which extend from the shoreline up to $x_{b}$ and have small alongshore wavelengths. Therefore we perform experiments 


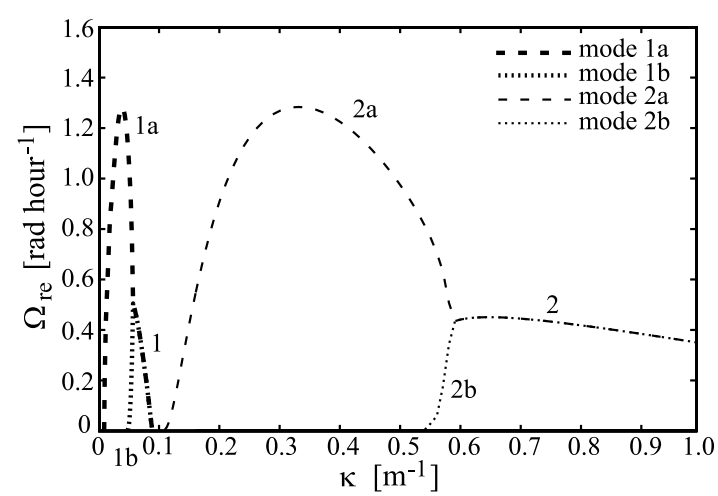

(a)

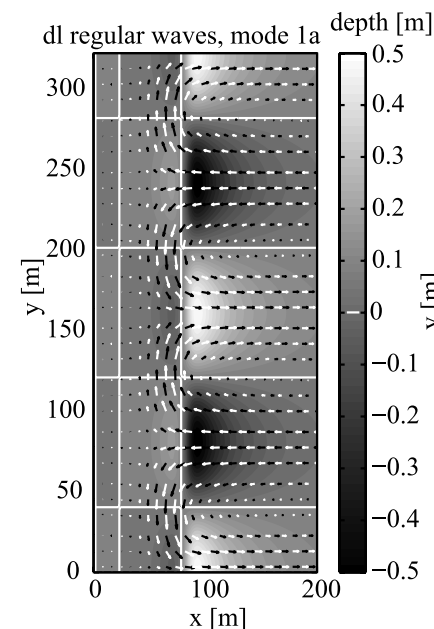

(b)

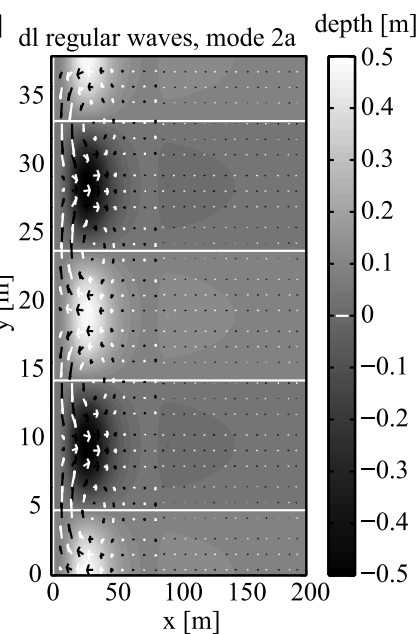

(c)
Figure 5. Results for depth-limited regular waves $(n=50$, $m=30$ ) including shoaling effects and wave refraction over the bed but excluding wave refraction over the current: (a) growth rate curve, (b) fastest growing pattern for $\kappa=0.039$ $\mathrm{rad} / \mathrm{m}$, and (c) fastest growing pattern for $\kappa=0.331 \mathrm{rad} / \mathrm{m}$. Note the different alongshore length scales in Figures $5 \mathrm{~b}$ and $5 \mathrm{c}$.

here that include wave refraction over the emerging bed as well as shoaling but exclude refraction over the emerging current (see Figure 5). As expected both a crescentic bar pattern and a transverse bar pattern are found. The crescentic bars extend to $\sim 200 \mathrm{~m}$ offshore and have large circulation cells. This is due to the omission of wave-current interaction [Yu and Slinn, 2003], as the rip currents are not opposed by the incoming wavefield. The smaller offshore extent of the crescentic bars, compared to results including only shoaling, is due to wave refraction over the bed. This focuses cross-shore momentum over the shoal [Caballeria et al., 2002], resulting in a shoreward directed (i.e., negative) force on the water column and thus onshore flow generation. As such it reduces the efficiency of the feedback mechanism caused by wave shoaling, leading to spatially reduced features.

[30] Transverse bars are located close to the shoreline and have a small alongshore wavelength $\left(\lambda=0.25 x_{b}\right)$. This is less than the value reported by Caballeria et al. [2002] $(\lambda=$ $0.5 x_{b}$ ), which may be due to the nonlinear effects incorporated in the latter study (influence of subharmonic modes and quadratic friction). Moreover the different sediment transport descriptions can have some influence as well. As before, growth is caused by the onshore flow over the shoal in the surf zone, where depth-averaged stirring increases offshore.

[31] For $H_{\infty}=1.0 \mathrm{~m}$ the two patterns exhibit an approximately equal growth rate (e-folding timescale $\sim 45 \mathrm{~min}$ ), which could result in a mixed pattern like those of Caballeria et al. [2002]. Numerical experiments with a lower $(0.5 \mathrm{~m})$ and higher $(1.5 \mathrm{~m})$ offshore wave height showed dominance of the transverse bar pattern for low forcing conditions and unclear results for high forcing conditions (no maximum growth rate was found for mode 2): see Caballeria et al. [2002]. Decreased wave forcing decreases both the morphological diffusivity $\gamma(x)$ and the stirring $\alpha(x)$, with the strongest decrease in $\gamma(x)$. This favors the growth of short wavelength features which are otherwise damped due to their steep slopes. High wave forcing would lead to dominant diffusivity and thus beach stability.

[32] When shoaling is excluded so that only wave refraction over the depth is accounted for the results show dominance of the transverse bar pattern for the default offshore wave height $H_{\infty}=1.0 \mathrm{~m}$. This dominance is due to a slight growth rate decrease for the transverse bar pattern; the crescentic bar pattern shows a much lower growth rate, similar to those predicted when refraction on

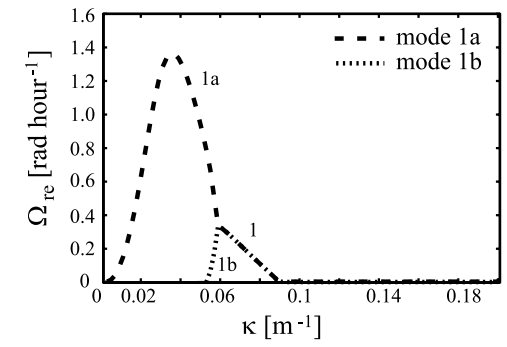

(a)

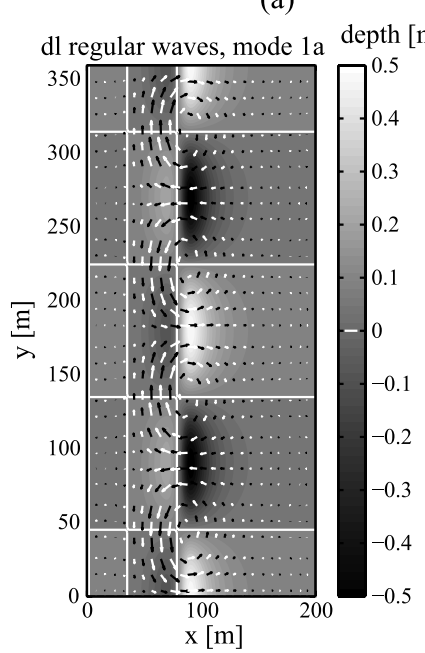

(c)

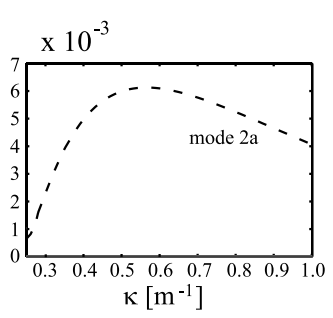

(b) depth $[\mathrm{m}]$

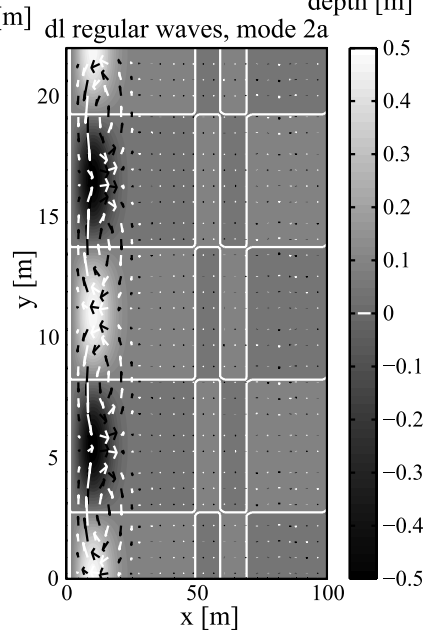

(d)

Figure 6. Results for depth-limited regular waves $(n=50$, $m=30$ ): (a) growth rate curve, (b) growth rate curve for larger $\kappa$, and bed perturbation with velocity field for fastest growing (c) mode $1(\kappa \sim 0.035 \mathrm{rad} / \mathrm{m})$ and (d) mode $2(\kappa \sim$ $0.55 \mathrm{rad} / \mathrm{m})$. Note the different alongshore length scales in Figures $6 \mathrm{~b}$ and $6 \mathrm{c}$. 


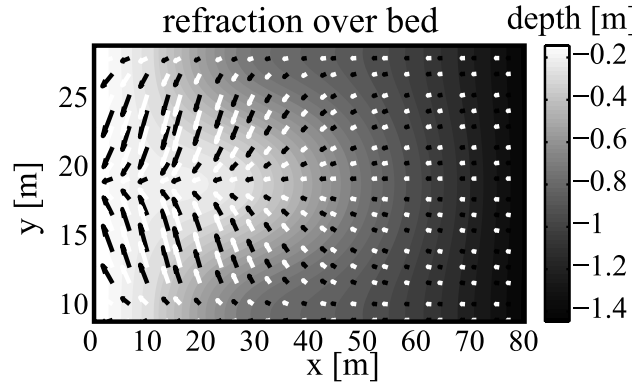

(a)

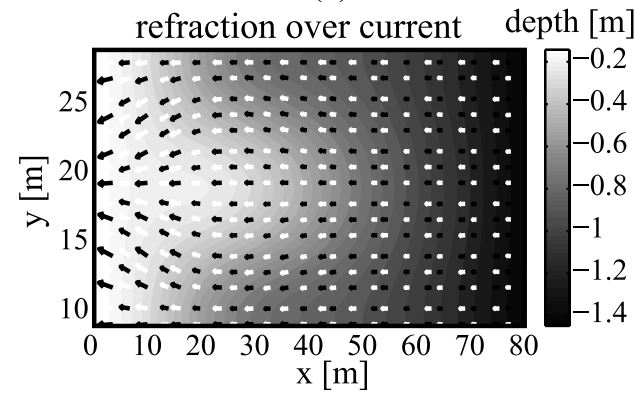

(b)

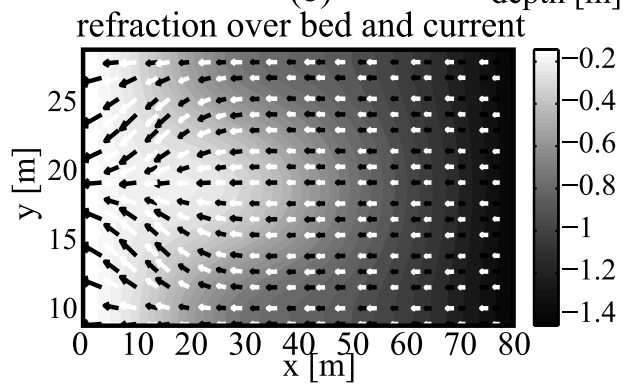

(c)

Figure 7. Total (equilibrium plus perturbation) wave number field (arrows) over depth (m) for depth-limited regular waves $(n=50, m=30)$ over a transverse bar (equilibrium plus perturbations): (a) including shoaling effects and wave refraction over the bed, (b) including shoaling effects and wave refraction over the current, and (c) including shoaling effects and wave refraction over both bed and current.

depth was also excluded (see Figure 4). The refraction effect over transverse bars is evidently acting to produce a strong positive feedback leading to their growth, whereas crescentic bars are largely unaffected by this.

\subsection{Influence of Wave-Current Interaction}

[33] When wave refraction over the current is subsequently reincorporated the full model equations are used (see Figure 6). The growth rate curve shows two modes, of which one has a relatively small growth rate and is shown in an enlargement. The fastest growing solution has an e-folding timescale $\sim 43 \mathrm{~min}$ and is a crescentic bar pattern (Figure 6c). This mode has a slightly larger alongshore wavelength and a more pronounced shoaling zone bed perturbation than that found when excluding shoaling and refraction (Figure 4b). Wave shoaling effects cause the observed seaward skew in intensity, as explained in the previous sections. The second mode is a transverse bar pattern (Figure $6 \mathrm{~d}$ ) and has an e-folding time of $\sim 6.8$ days. Experiments with a smaller offshore wave height $(0.5 \mathrm{~m})$ using the full equations showed similar results, i.e., strong dominance of the crescentic bar pattern. When refraction over the bed is excluded the transverse bar solution disappears, leaving the crescentic pattern as the only mode, with a reduced positive growth rate of 1.1 hour $^{-1}$.

[34] To study the growth and decay of the transverse bar pattern in more detail, a flow over topography (FOT) experiment is performed. The transverse bar bed pattern of Figure $5 \mathrm{c}$ is assumed fixed, and the corresponding hydrodynamics are calculated (see Figure 7). In all cases the bed pattern will generate an offshore flow over the shoal where $\frac{\partial h}{\partial x}>0$ and an onshore flow where $\frac{\partial h}{\partial x}<0$, due to greater breaking over the shoal (as explained before). Similarly, in all cases the wavefront is focused over the shoal, but the effect is clearly stronger for the case including wave refraction over depth only (Figure 7a). Here the onshore flow over the entire shoal caused by refraction is dominant over the offshore flow near the shore generated by the local reduction in depth (see Falqués et al. [2000] and section 4.2) resulting in a net onshore flow (see Figure $5 \mathrm{c}$ ) and growth of the feature. When only shoaling and wave refraction over the current are accounted for (Figure $7 \mathrm{~b}$ ) the bed pattern will generate an offshore flow over the shoal where $\frac{\partial h}{\partial x}>$ 0 and an onshore flow where $\frac{\partial h}{\partial x}<0$, as explained before. As the incoming wavefield encounters the offshore current over the shoal the wavefront is decelerated. The opposite occurs in the trough areas where the wavefront is locally accelerated, resulting in a focusing of crossshore momentum over the shoal and a weak onshore flow. However, this effect is not strong enough to counteract the original offshore current, resulting in a net offshore directed current and decay of the feature. Figure $7 \mathrm{c}$ shows the wave number field calculated using the full model equations. Here the refraction over the bed produces an onshore flow over the entire shoal which is dominant over the offshore flow generated due to the decreasing depth. However, wave refraction over the onshore current accelerates the local wavefront over the shoal, while it decelerates the flow over the offshore current in the troughs. This causes a focussing of crossshore momentum over the troughs, thus weakening the effect of refraction over the bed. The net result is a weak onshore current over the shoal and thus slow growth of the bed pattern.

\subsection{Overview}

[35] The results of the different model experiments presented above are summarized in Figure 8. Clearly, the transverse bar pattern depends on the presence of wave refraction over the bed, whereas crescentic patterns experience a positive feedback from all mechanisms. As the effect of wave refraction over the current counteracts the positive feedback from refraction over depth for tranverse bars, the most likely pattern to be observed in nature is the crescentic bar pattern.

\subsection{Near-Normal Incidence}

[36] Last, using the full model equations we find three modes for small angles of wave incidence. The dominant 


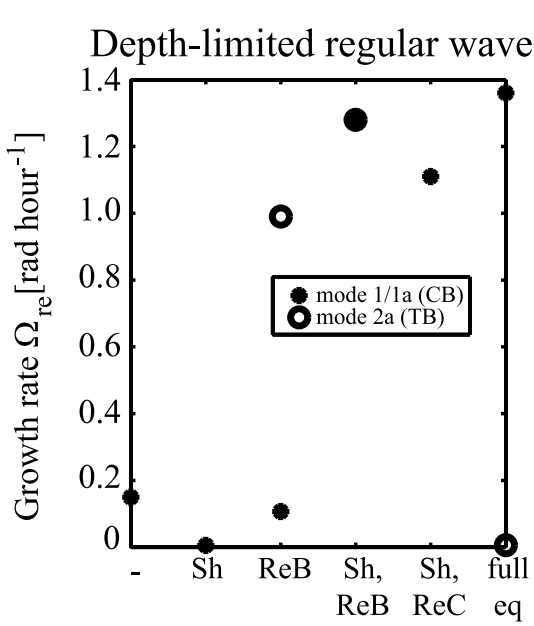

Physics involved

(a)

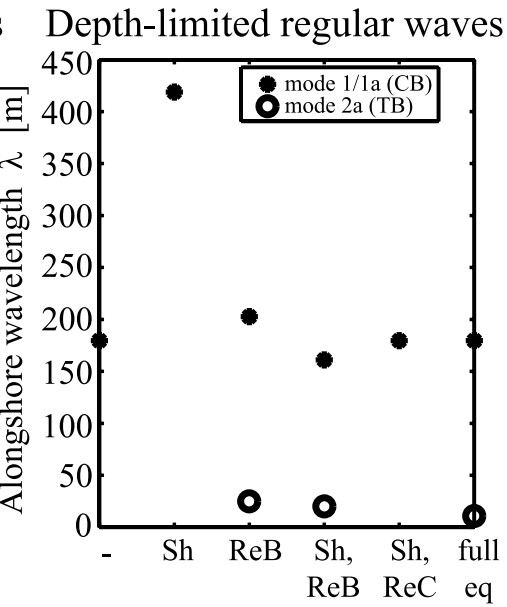

Physics involved

(b)

Figure 8. Results for depth-limited regular waves obtained when including different physical feedbacks: (a) dominant growth rate and (b) preferred alongshore wavelength. Here Sh stands for wave shoaling, ReB stands for wave refraction over the bed, and $\mathrm{ReC}$ stands for wave refraction over the current. The minus sign refers to exclusion of $\mathrm{Sh}, \mathrm{ReB}$, and $\mathrm{ReC}$ (approximately the Falqués model), whereas the full equations include $\mathrm{Sh}, \mathrm{ReB}$, and $\mathrm{ReC}$.

mode (mode 1) is similar to the crescentic bar feature shown in Figure 6c, while the third mode resembles the transverse bar pattern of Figure $6 \mathrm{~d}$. The second mode is a crescentic bar pattern with elongated inner shoals and troughs under an oblique angle and is shown in Figure 9. All modes migrate in the downstream direction (the alongshore current flows in the positive $y$ direction for positive incidence angles) with a rate of $86 \mathrm{~m} /$ day, $130 \mathrm{~m} /$ day and $0.08 \mathrm{~m} /$ day for mode 1,2 and 3 , respectively. The second mode resembles those found by Ribas et al. [2003] (their mode A). Contrary to their results a similar mode with opposite pattern direction in the surf zone is not found. Here mode 1 and 2 converge for normal incidence and for $0.06<\kappa<0.09$ to form the double mode shown in Figure 6a. This indicates that the complex conjugate solutions should be considered as separate modes which can evolve each one independently of the other. The next section will look in more detail at double mode solutions.

\section{Bed Pattern Generation for Regular to Random Waves}

[37] Here we progressively relax the assumption of regular, depth-limited waves, by applying different wave dissipations: see section 3. Results are shown for experiments using the full model equations, unless clearly stated otherwise.

\subsection{Regular Waves}

[38] The linear stability results for regular waves (not depth-limited) are very similar to those obtained for depthlimited regular waves as presented in section 4 (see Figure 6). This similarity is due to a natural depth limitation of the wave height in the surf zone, as shown in Figure 2 and explained in section 3 .

\subsection{Intermediate Waves}

\subsubsection{Growth Rate and Bed Pattern Results for the} "Falqués" Model

[39] In Figure 10 we show the growth rate curve and dominant bed patterns for intermediate waves. See Figure 2 for the cross-shore equilibrium profiles. The results show that the range of alongshore wave numbers which allows for

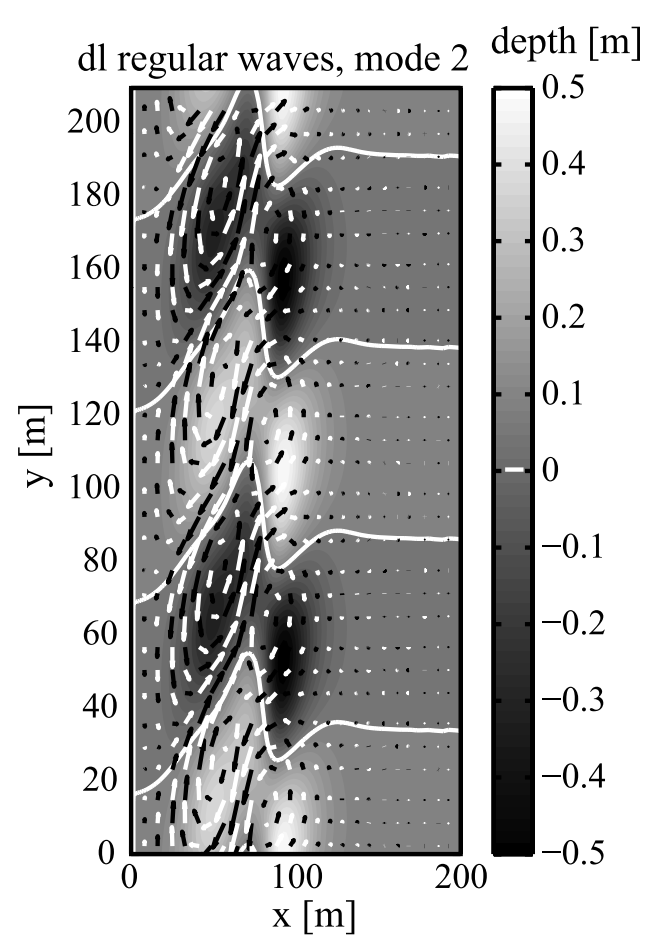

Figure 9. Results for depth-limited regular waves $(n=50$, $m=30$ ): mode 2 perturbation bed pattern. 


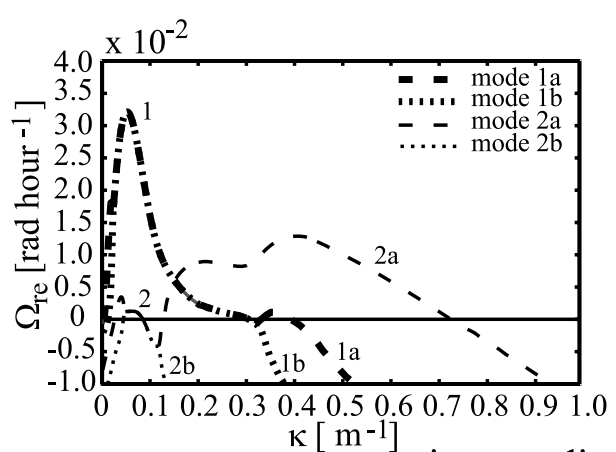

(a)

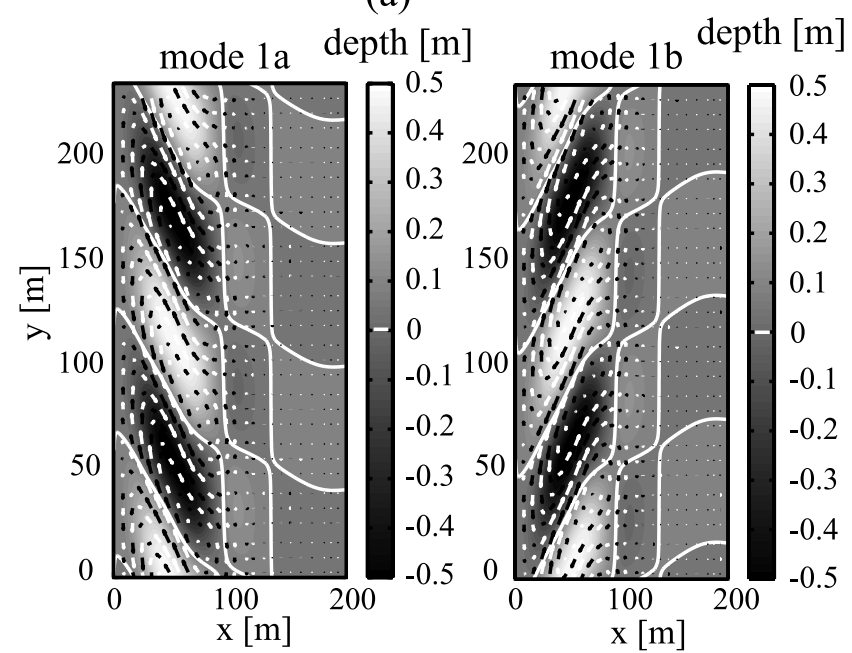

(b)

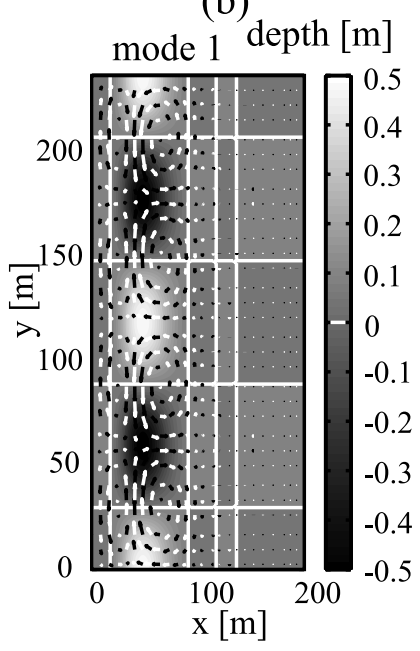

(d) (c) depth [m]

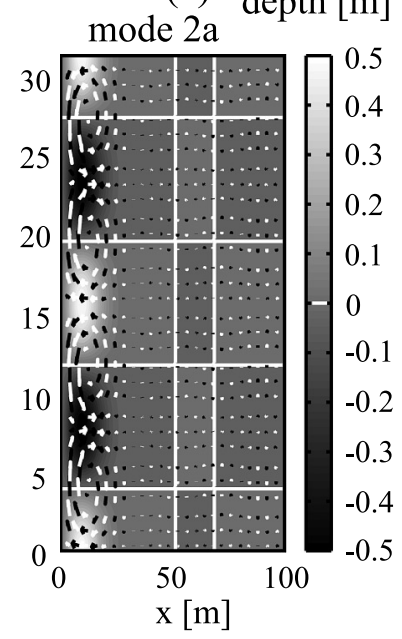

(e)
Figure 10. Results for intermediate waves $(n=10, m=0$, $\gamma_{b}=0.55$ ): (a) growth rate curve, (b) dominant bed pattern for $\kappa=0.053 \mathrm{rad} / \mathrm{m}$, (c) complex conjugate of dominant bed pattern for $\kappa=0.053 \mathrm{rad} / \mathrm{m}$, (d) the pattern obtained by superposition of patterns in Figures $10 \mathrm{~b}$ and $10 \mathrm{c}$, and (e) dominant bed perturbation for $\kappa=0.41 \mathrm{rad} / \mathrm{m}$. Note the different alongshore length scales.

complex conjugate solutions (double mode) has strongly increased. Bifurcation behavior is observed as mode 1a and $1 \mathrm{~b}$ become complex conjugates (referred to as mode 1 ) for $\kappa=0.023 \mathrm{rad} / \mathrm{m}$ and split again for $\kappa=0.33 \mathrm{rad} / \mathrm{m}$. The same is observed for a second mode, mode 2 , which shows a maximum for $\kappa=0.41 \mathrm{rad} / \mathrm{m}$ (mode $2 \mathrm{a}$ ). The maximum growth rate is for mode 1 , consisting of oblique bars with opposite orientation, see Figures $10 \mathrm{~b}$ and $10 \mathrm{c}$. Here these double modes evolve at the same rate, yielding the bed pattern shown in Figure 10d. The time evolution of this superimposed pattern would be oscillatory. However, as pure normal wave incidence does not occur in nature it is more likely that a mixed pattern would be observed. The pattern corresponding to the maximum of mode $2 \mathrm{a}$ is shown in Figure 10e and is a transverse bar pattern located close to the shore. Both double modes are characterized by a pattern shift from crescentic bars to transverse bar for increasing $\kappa$. This seems natural as steeper alongshore slopes induce a stronger smoothing due to morphological diffusion. As this diffusion is largest around the breakpoint the pattern is slowly forced into the nearshore zone. The main difference with regular waves is that these nearshore patterns now exhibit positive growth rates.

[40] The growth rate of the dominant mode for normal wave incidence has decreased to an e-folding time $\sim 1.3$ days. This is due to spreading of wave breaking and a lower breaker index, causing a reduction in depth-averaged stirring. All bed patterns are prominent in the surf zone. Note also, however, that the seaward part of modes $1 \mathrm{a}$ and $1 \mathrm{~b}$ in Figures $10 \mathrm{~b}$ and $10 \mathrm{c}$, i.e., that seaward of $x_{b} \approx 90 \mathrm{~m}$, is contained within the region where the dissipation is nonzero (or significantly so). This implies that dissipation prior to maximum breaking is having an effect. Analysis of the different terms in the phase equation (4) shows that wave refraction over depth is important in this region, whereas for (depth-limited) regular waves this contribution was negligible compared to that of wave refraction over the current. Here dissipation offshore of the breakpoint reduces wave shoaling, effectively reducing the strength of the offshore current due to shoaling. This effect will be even more pronounced for fully random waves. The terms in the bottom evolution equation show that the bed perturbation is driven by the cross-shore current term (coupled with stirring): see Falqués et al. [2000]. Other terms reflect the balance in the continuity equation.

[41] The double mode solutions split into two separate growth rate curves for small incidence waves with the down-current-oriented bars (Figure 10c) becoming the dominant pattern. Down current is defined here as the position of the seaward part of the bar with respect to its shoreward part.

\subsubsection{Influence of Wave Shoaling and Refraction}

[42] A similar analysis to that presented for depth-limited regular waves (see section 4) was carried out to investigate the separate effects of wave shoaling and wave refraction over depth or current using intermediate waves (see Figure 11).

[43] The main results support the previous observations for depth-limited regular waves, i.e., wave refraction over the bed strongly favors transverse bar growth and wave refraction over the current strengthens crescentic bar growth but opposes transverse bar formation. The results for mode 1a and 2a were not incorporated unless the growth rate curve showed a local maximum corresponding to a transverse bar pattern. However, remarkably, when the reduced model equations (excluding shoaling and refraction in the 


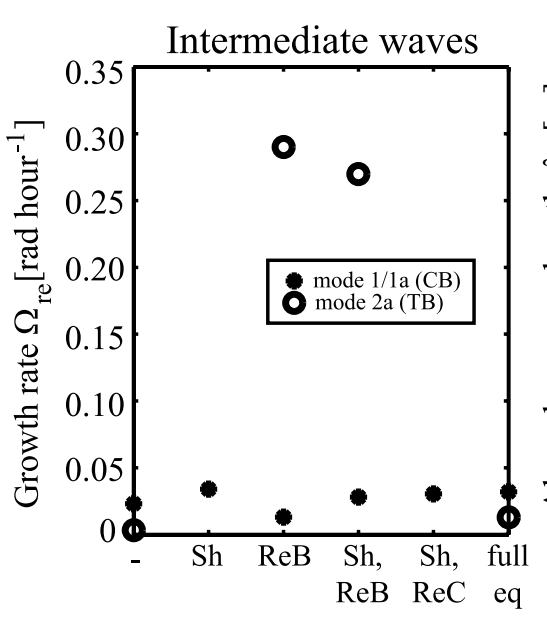

Physics involved

(a)

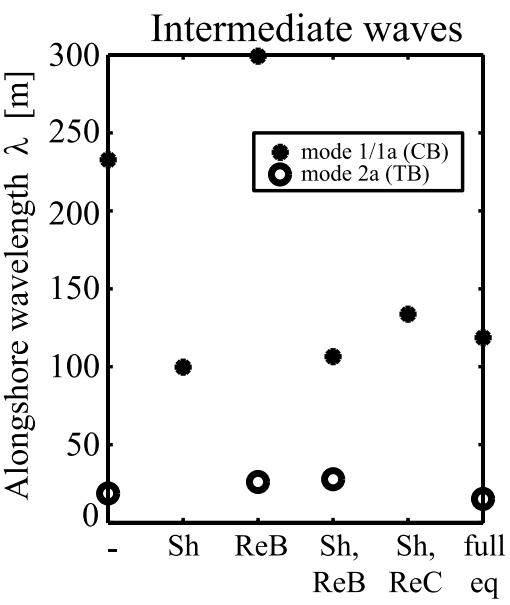

Physics involved

(b)

Figure 11. Results for intermediate waves obtained when including different physical feedbacks: (a) dominant growth rate and (b) preferred alongshore wavelength. See Figure 8 for an explanation of the symbols.

perturbations) were forced with intermediate waves, both a crescentic bar pattern and a transverse bar pattern were found. The crescentic pattern is similar to that shown in Figure 10b, while the transverse bar pattern is shown in Figure 12. This pattern is characterized by a maximum in the bed perturbation located very close to shore. Without shoaling and refraction the basic feedback mechanisms are those related to the moving break point and differential breaking due to reduced depth. The transverse bars are located very close to the shore, indicating that the first mechanism can be disregarded. The second mechanism will be modified as the waves are no longer depth-limited. However, we assume here that this modification will be small compared to the basic balances. The reduced equilibrium depth near the shore for intermediate waves causes a change in balance in the perturbation cross-shore momentum equation. With a similar wave height the energy contribution to the radiation stress term remains unchanged (compared to depth-limited regular waves with an equal breaker index), whereas the depth contribution increases due to the decreased equilibrium depth. This leads to the generation of an onshore current in order to retrieve the balance. This current is onshore directed over the entire shoal as the bed perturbation is contained in the region where $H / D$ increases. The onshore current generated by this increases is strong enough to overcome a weak offshore directed current generated by differential breaking, causing growth of a skewed pattern with a limited region of $\frac{\partial h}{\partial x}>0$. A more detailed analysis showed that this transverse bar pattern also occurs for regular waves (not depth limited) using the reduced equations. However, due to the small region of $H / D>\gamma_{b}$ the pattern has a large e-folding time $\sim 41$ days and is therefore not expected to appear in nature. A similar result is predicted for random wave forcing. Other results show a strong increase in extent of the $\kappa$ region where double mode solutions occur when wave shoaling is added to the model. An increase in growth rate is observed in this case, whereas depth-limited regular waves showed a decrease in $\Omega_{r e}$.

\subsection{Fully Random Waves}

[44] In Figure 13 we show the growth rate curve and predicted bed pattern for fully random waves. Again, a double mode is found for a range of alongshore wave numbers, including the dominant wave number. The dominant bed pattern resembles an oblique bar system with the bed perturbations extending from the shoreline up to the location of maximum depth-averaged wave stirring. When these complex conjugate patterns (Figures 13b and 13c) are

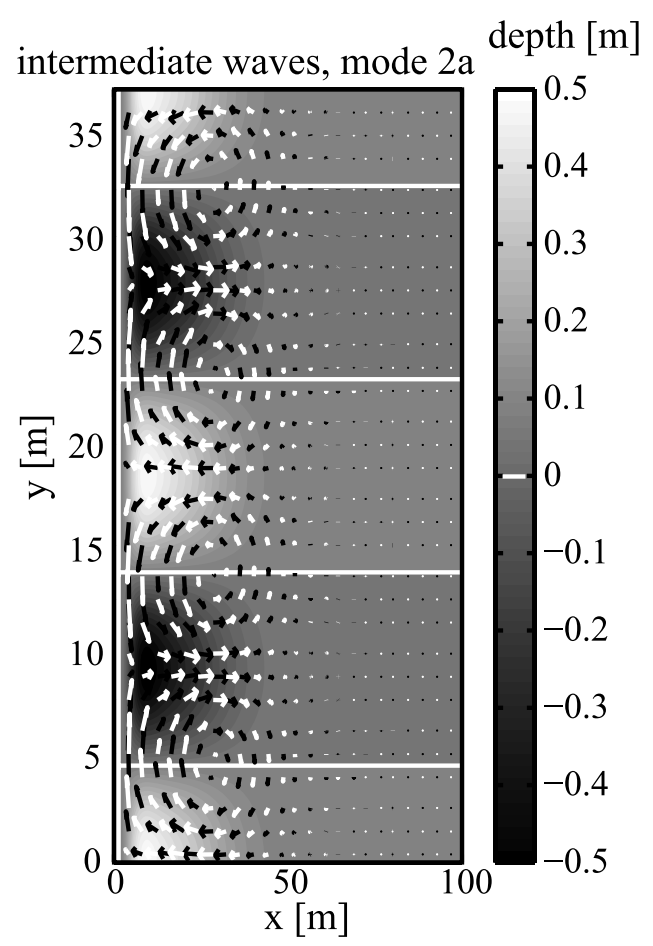

Figure 12. Results for intermediate waves $(n=10, m=0$, $\gamma_{b}=0.55$ ): transverse bar pattern for reduced equations (no wave shoaling or refraction effects). 


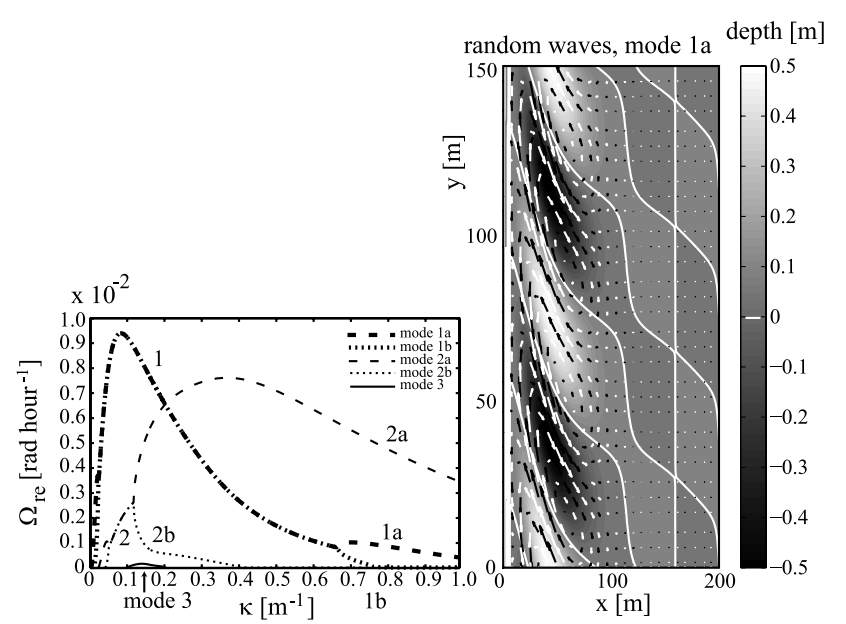

(a)

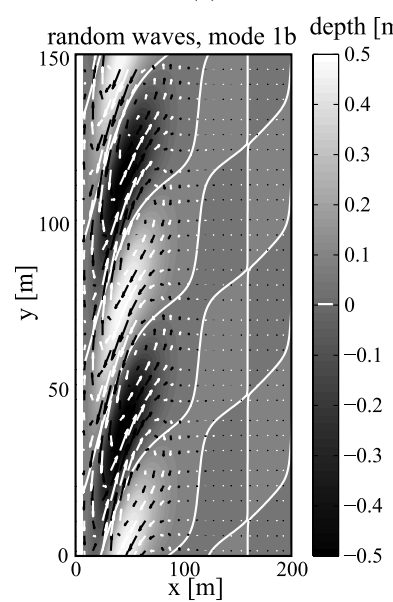

(c) (b)

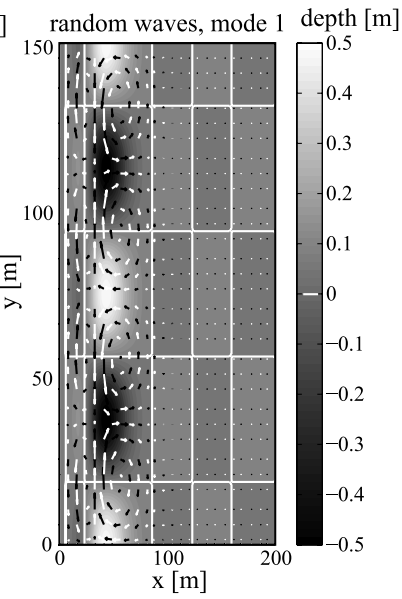

(d)
Figure 13. Results for random waves: (a) growth rate curve, (b) dominant bed pattern, (c) complex conjugate of dominant bed pattern, and (d) dominant bed pattern if both modes are superimposed. Default forcing values are $H_{r m s, \infty}=1.0 \mathrm{~m}, T_{p, \infty}=6 \mathrm{~s}$, and $\theta_{\infty}=0^{\circ}$.

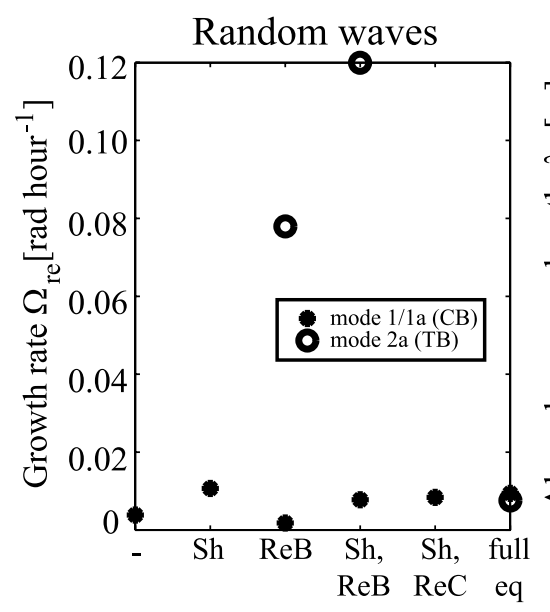

Physics involved

(a) combined the pattern shown in Figure 13d is obtained. This pattern shows a mirroring effect near the shoreline and double circulation cells. The e-folding time is $\sim 106$ hours or 4.4 days. This is a considerably slower growth than that found for regular waves, due to the lower breaker index and spread dissipation. The wavelength $\lambda \sim 75 m \sim 0.6 x_{b}$ corresponds to the $\lambda=0.5 x_{b}$ found by Caballeria et al. [2002] for transverse bars. The second mode is again characterized by a maximum for the single mode $2 \mathrm{a}$. The pattern is a transverse bar similar to the one shown in Figure 10e. Without significant wave shoaling effects shoaling zone patterns are absent. Bed patterns evolve in the surf zone between the shoreline and the maximum in depth-averaged stirring, with a possible mirroring effect shoreward of this location. When a small wave angle is imposed $\left(\theta_{\infty}=1^{\circ}\right)$ the double modes split and the down-current-oriented pattern becomes dominant over the up-current-oriented one.

\subsubsection{Wave Shoaling and Refraction Effects}

[45] We show growth rates for the different feedback mechanisms in Figure 14. Here the results for mode 2 were not incorporated unless the growth rate curve showed a local maximum corresponding to a transverse bar pattern. Although the patterns in Figure 13 are all surf zone patterns, analysis of the separate influences of wave shoaling and refraction shows similar results to those obtained before: wave refraction over current favors growth of mode 1, while wave refraction over the bed stimulates growth of mode 2 . Thus the oblique bars depend on a different feedback mechanism from the transverse bars. When the reduced model was applied (excluding shoaling and refraction effects) growing transverse bar patterns were observed with such a slow growth rate (corresponding to $\sim 19$ years) that they are considered nonexisting. Note, finally, that for random waves the growth rates for crescentic and transverse bars are almost the same: see Figure 14.

\subsubsection{Variation in Offshore Wave Height and Period}

[46] As the dominant bed pattern seems to depend on the amount of offshore dissipation, numerical experiments are performed for different wave heights and periods (see

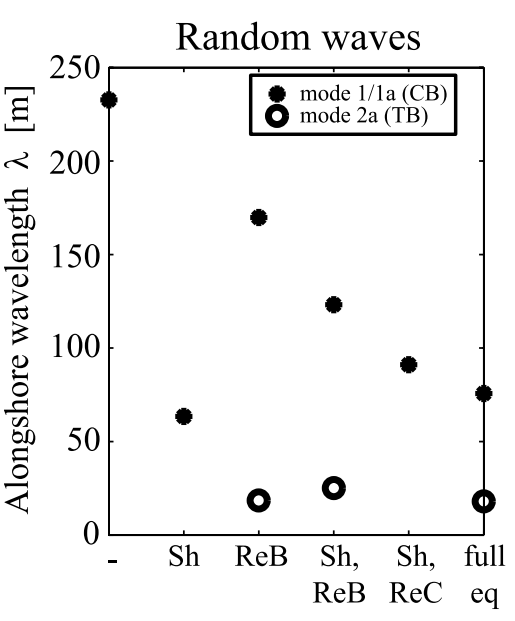

Physics involved

(b)

Figure 14. Results for random waves obtained when including different physical feedbacks: (a) dominant growth rate and (b) preferred alongshore wavelength. See Figure 8 for an explanation of the symbols. 


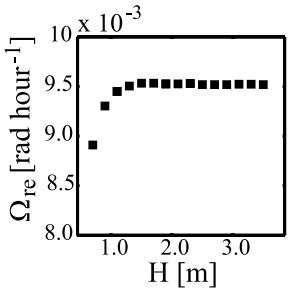

(a)

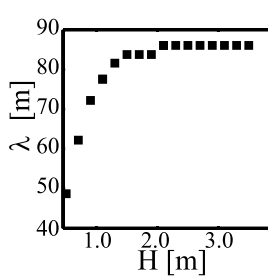

(b)

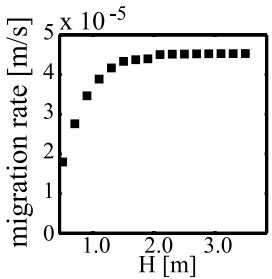

(c)
Figure 15. Results for random waves with varying offshore wave height: (a) growth rate, (b) wavelength, and (c) migrating rates for the fastest growing mode. Default values include $T_{p, \infty}=6 \mathrm{~s}$ and $\theta_{\infty}=0^{\circ}$.

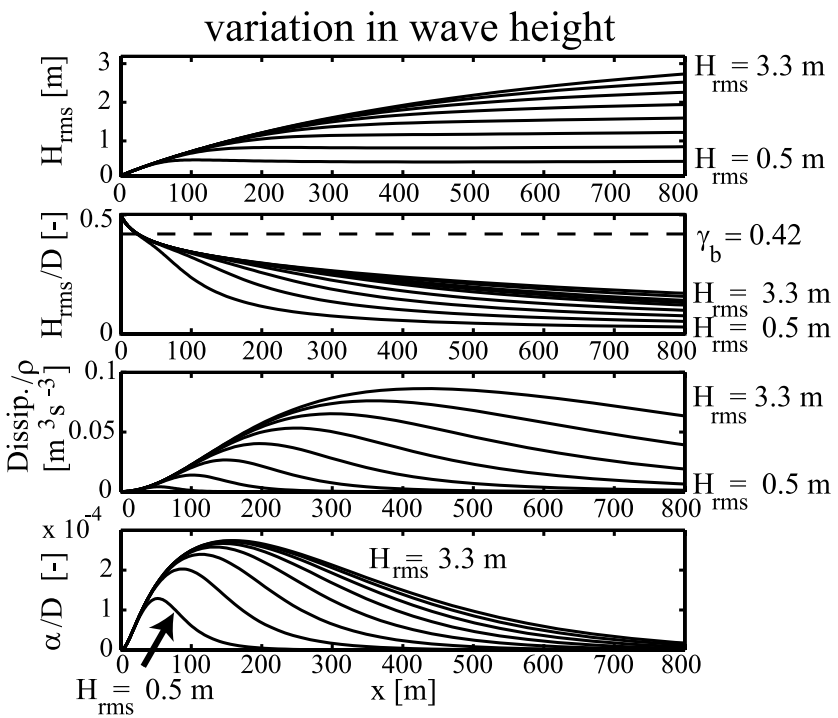

(a)

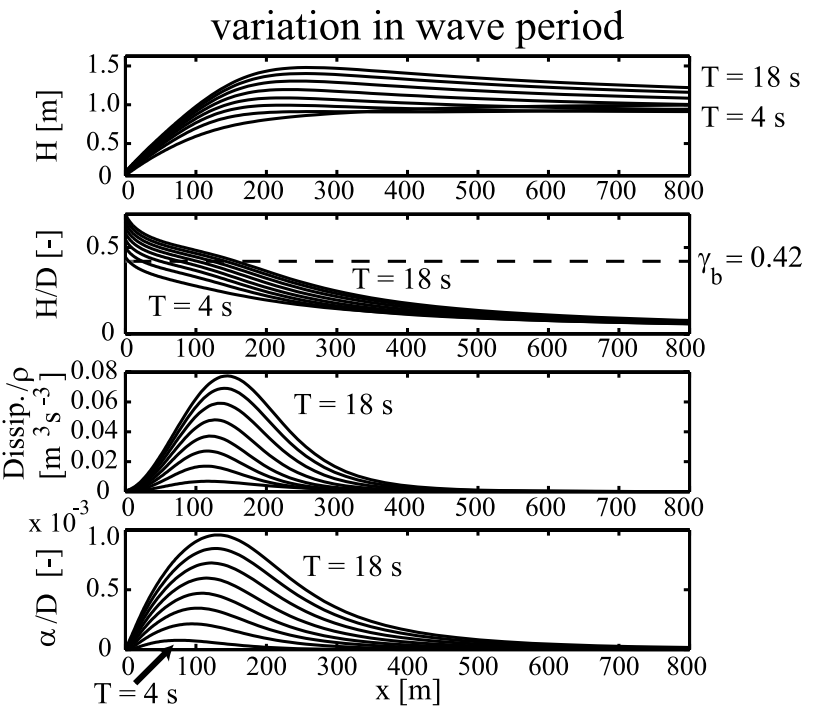

(b)

Figure 16. Cross-shore profiles for random waves (normal incidence) and (a) varying offshore wave height $(0.5--3.5$ $\mathrm{m}$ with increments of $0.2 \mathrm{~m}, T_{p, \infty}=6 \mathrm{~s}$ ) and (b) varying offshore wave period (4--18 s with increments of $1 \mathrm{~s}$, $\left.H_{r m s, \infty}=1.0 \mathrm{~m}\right)$.
Figure 15). For all values of $H_{r m s, \infty}$ applied here a double mode was found as the dominant pattern. The results show a saturation in alongshore wavelength and migration rate for higher values of the offshore wave height forcing. This is due to a saturation in the maximum of the depth-averaged wave stirring function (see Figure 16a). The cross-shore extent of the pattern saturates at a distance of approximately $100 \mathrm{~m}$ due to saturation of the offshore wave height caused by the increased dissipation offshore for large wave heights. The saturation is therefore a direct result of the imposed random dissipation, and was not observed for regular wave forcing.

[47] Saturation does not occur for increasing wave periods (see Figure 16b). The maximum in depth-averaged stirring increase and moves offshore. The position of maximum dissipation also moves offshore for increasing periods, but dissipation seaward of this location does not increase significantly. Therefore there is no saturation in preferred wavelength or growth rate for random waves with increasing period (see Figure 17).

\section{Discussion of the Results \\ 6.1. Regular Versus Random Waves}

[48] The results for (depth-limited) regular, intermediate and random waves show marked differences. The regular wave case (for both depth-limited waves and otherwise) focuses wave breaking at one point, causing large energy dissipation there. The result is a strong peak in sediment available for transportation at this location and a high growth rate for the growing bed pattern. Both a crescentic and a transverse bar pattern are found, but, with respective e-folding times of $45 \mathrm{~min}$ and 6.8 days.

[49] The intermediate case showed the existence of two double (i.e., consisting of complex conjugates), growing modes, with the dominant mode consisting of two oblique crescentic bar patterns with elongated surf zone parts. The second mode showed a local maximum for higher values of the alongshore wave number, corresponding to a transverse bar pattern. The transverse bar pattern showed a reduced growth rate compared to the oblique crescentic bars. With similar cross-shore locations the transverse bar pattern is not expected to appear in nature.

[50] Random waves showed a spread dissipation (as expected) and the presence of again two double, growing modes. The dominant double mode consisted of two

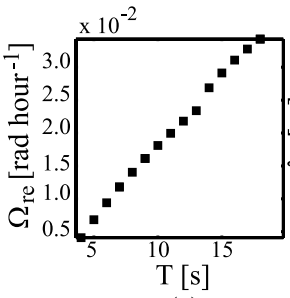

(a)

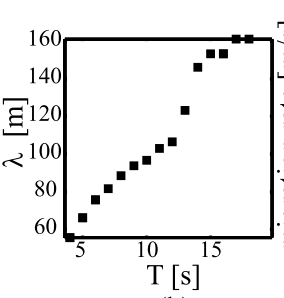

(b)

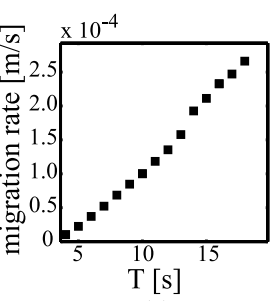

(c)
Figure 17. Results for random waves with varying offshore wave period $\left(H_{r m s, \infty}=1.0 \mathrm{~m}\right.$ and $\left.\theta_{\infty}=0^{\circ}\right)$ : (a) growth rate, (b) wavelength, and (c) migrating rates for the fastest growing mode (propagating in the positive alongshore direction). 

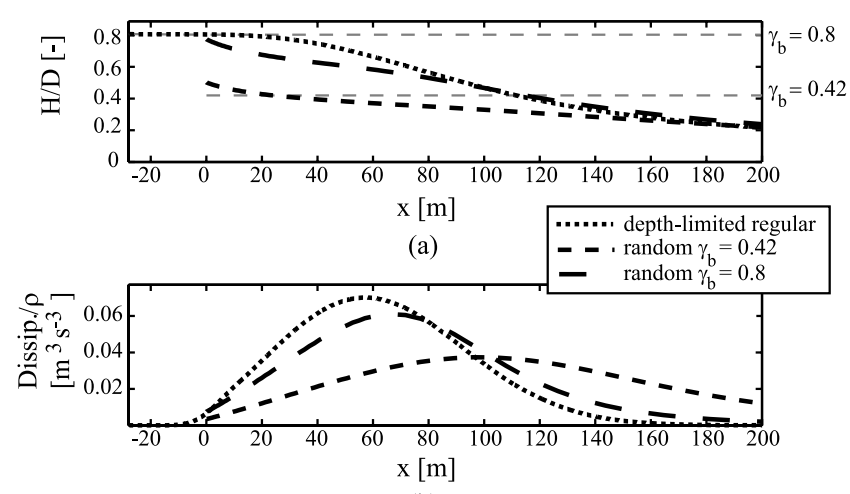

(b)

Figure 18. Cross-shore profiles of (a) wave height over depth ratio and (b) dissipation for regular and random wave forcing.

oblique bar patterns. The second double mode showed a maximum for large $\kappa$, and was a nonmigrating, transverse bar pattern with a growth rate similar to the dominant double mode. Sediment is available throughout the surf zone with no clear maximum but with decreasing availability near the shoreline and offshore. Thus the predicted bed patterns are located in the surf zone or close to the shoreline. The amount of energy close to the shore is significant in this case. A mixed pattern would be expected to appear in nature, with characteristics of transverse and oblique bars. Growth rate values found here are not uncommon in the field [see van Enckevort et al., 2004].

[51] Thus transformation of wave height in surf and shoaling zones largely controls the cross-shore extent of the bed features that can develop, depending on the energy available to move sediment in this region. Results here show that patterns with a strong offshore profile (maximum bed perturbation offshore of $x_{b}$ ) can develop in more regular wave regimes, or regimes with less wave energy close to the shore. The former may, perhaps, be due to swell dominated conditions; the latter can depend strongly on beach slope, with a large slope giving much energy near the shore. Patterns with a dominant surf zone profile may be found for more random wave climates.

[52] The data dependence of the random wave expression by Thornton and Guza [1983] is further investigated with a statistical experiment. The cross-shore profiles of a large number of regular waves $(100,000$ waves with wave heights following a Rayleigh distribution) are averaged for comparison with random wave results (using $H_{r m s}$ of the same distribution). Figure 18 shows the resulting dissipation profiles. Clearly, the lack of field data information in the regular wave profile prohibits a good match. Even when equal breaker indices are applied the random wave model still shows an offshore shift in the location of maximum dissipation. When the field-based breaker index of $\gamma_{b}=0.42$ is applied the location of maximum dissipation moves even further offshore while the magnitude decreases significantly. This implies that great care must be taken when comparing results obtained with regular wave forcing (as by Falqués et al. [2000], Caballeria et al. [2002], and Ribas et al. [2003]) with those obtained with random wave forcing (as by Calvete et al. [2005]).

\subsection{Migrating Patterns for Normal Wave Incidence}

[53] The double mode solution, consisting of two modes which are complex conjugates, has been a persistent feature of our model experiments. These patterns display equal growth and migration rates but migrate in opposite directions and are characterized by surf zone patterns in opposite alongshore directions. These patterns should be viewed as individual modes, each capable of growth independent of the other. Normal incidence yields equal growth rates; for oblique incidence the two modes separate and become two different patterns with different growth rates. More random wave forcing favors the occurrence of double mode solutions.

[54] To further examine the presence of double mode solutions different beach slopes were applied. All slopes presented here lead to saturated conditions in the surf zone. Results for fully random waves are shown in Figure 19 and indicate that the transverse bar pattern for higher alongshore wave numbers is favored by mild beach slopes. With a slope of $\beta=0.05$ the transverse pattern is no longer present as a growing mode. As beach slope increases, there is a decrease in the range of alongshore wave numbers that allow a crescentic bar pattern (mode 1). For beach slopes steeper than 0.04 the crescentic pattern corresponding to the local maximum in growth rate for small $\kappa$ (see Figure 13a) becomes dominant. This is again a (nonmigrating) single mode solution.

[55] Calvete et al. [2005] considered the barred beach profile of Yu and Slinn [2003] (based on measurements from Duck, North Carolina, USA). They did not find a complex conjugate solution, although the present model was applied. Section 5.3.2 already explored the occurrence of double modes for larger periods, so the main remaining difference is the imposed bed profile. Therefore experiments were performed with different beds to bridge the gap between the present planar profile and that of Yu and Slinn [2003]. First, an exponential profile was imposed with a nearshore slope equal to that of the planar beach $(\beta=0.02)$ but exponentially decaying offshore until a steady depth of $20 \mathrm{~m}$. The results were nearly identical to those for the planar beach. A bar was then added to this exponential profile, resulting in decay of the transverse bar and increased growth rate of the crescentic pattern (water depth at the bar was $0.5 \mathrm{~m}$ ).

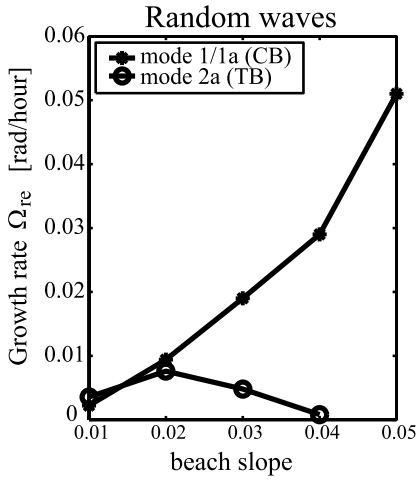

(a)

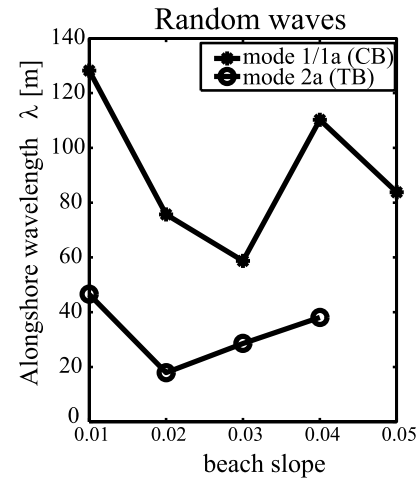

(b)
Figure 19. Growth rate results using fully random waves for different slopes on a plane beach: (a) growth rate and (b) alongshore wave number for the two different modes. 
However, the dominant crescentic pattern was now a single mode and the growth rate curve showed a strong decrease in the range of $\kappa$ that allows for double mode solutions. Different beach slopes applied here (i.e., increased depth over the bar) lead to different growth rate curves but always a double mode presence. Finally, the Duck beach profile showed solely single mode solutions, of which one was a tranverse bar pattern (growth rate $\sim 15$ hours, bar height similar to previous experiment but water depth over the bar was $2.0 \mathrm{~m}$ ). This profile is characterized by a steeper beachfront slope $(\beta>0.05)$ and a milder seaward slope (offshore of the bar) than the other profiles. Thus we hypothesize that a slope difference between the shoaling zone and bar region (where crescentic bars develop) and the inner surf zone (where tranverse bars are found) leads to separately operating feedback mechanisms for both patterns and therefore separate growth rate curves. The presence of a bar also enhances this separation, significantly reducing the occurrence of the double mode solution.

\subsection{Conditions Leading to Wave Energy at the Shore}

[56] In the past bed patterns have sometimes been attributed to the presence of infragravity motions [see, e.g., Holman and Bowen, 1982]. Our results have shown that the amount of wave energy near the shore has a significant impact on the type of bed form that can develop. Random wave forcing favors energy near the shore, as does a steep beach slope, the former because smaller waves penetrate further in without breaking and the latter because the waves have no time to adjust to the local depth. That high amount of wave energy near the shore causes a wave stirring distribution increasing shoreward. A similar wave stirring distribution could also be a consequence of significant lowfrequency wave energy. Even if our model does not include low-frequency waves we could speculate via the stirring distribution that a significant low-frequency wave energy fraction can also contribute to this shoreline energy and therefore to the generation of transverse bars. Thus plane beaches that exhibit edge wave activity might be expected also to exhibit transverse bar patterns over crescentic bar patterns. Edge and leaky wave activity is often associated with fairly steep plane beaches [see, e.g., Oltman-Shay and Guza, 1987]. Our results indicate that it is possible that the shoreward increase in stirring leads to a morphohydrodynamical feedback mechanism that will be particularly operative for steep beaches (whether through the presence of low-frequency wave energy or because of the inherent beach steepness and thus the lack of adjustment time for the waves).

\section{Conclusions}

[57] A comprehensive linear stability model has been applied to study pattern formation in the nearshore zone on a planar beach. A new expression was used to describe regular wave breaking throughout the cross-shore domain without the need of matching conditions at the breaker line. Results obtained with this new expression were compared to those obtained using the random wave dissipation expression of Thornton and Guza [1983]. The new expression for regular wave dissipation is a useful tool to control the wave height and thus the amount of energy within the surf zone.
[58] The results showed that different patterns are predicted for regular, intermediate and random waves on mild slopes. Thus the imposed depth-limited wave condition has a profound impact on the predicted morphology. This is particularly true as crescentic bars are found to be dominant for regular wave forcing using the model, whereas different patterns (resembling oblique, crescentic bars and transverse bars) with equal-order growth rates are found for more random wave forcing.

[59] The hypothesis of Niederoda and Tanner [1970] and subsequent conclusion by Caballeria et al. [2002] that wave refraction over depth causes transverse bar growth is further supported here. However, a positive feedback was also observed for non-depth-limited waves without refraction over depth. Thus it is concluded that both the non-depth-limited wave effect (increase in wave height over depth ratio in the surf zone) and wave refraction over depth cause transverse bar growth, with the latter mechanism possessing the strongest feedback. Wave refraction over the current opposes the feedback of wave refraction over depth for transverse bars. This effect was dominant for regular waves, leading to very slow growth rates for transverse bars when both feedbacks (wave refraction on depth and current) were included. When depth-limited waves were not assumed (intermediate and random waves) growth rates for crescentic and transverse bars were approximately equal. The spread of dissipation in these cases leads to longer e-folding times and weaker circulation cells.

[60] Results further showed that the existence of migrating patterns (double modes) for normal wave incidence on a planar beach is a very robust feature, particularly for more random wave forcing. This solution consists of two (independent) patterns that have opposite alongshore orientation and migration rates but equal growth rates. Numerical experiments with small incidence angles showed that these correspond to up- and down-current patterns when a weak alongshore current is present. Any configuration which allows both up- and down-current patterns to develop can therefore be expected to show double mode behavior for normal incidence. As pure normal incidence does not occur in nature a mixed pattern of the up- and down-current patterns can be expected in the field under near-normal wave conditions.

[61] Acknowledgments. We gratefully acknowledge the financial support from the UK Engineering and Physical Sciences Research Council (EPSRC) for S.M. van Leeuwen under grant GR/S19172/01. Partial funding by the Ministerio de Ciencia y Tecnologia of Spain through the PUDEM project under contract REN2003-06637-C02-01/MAR and through the "Ramón y Cajal" contract of D. Calvete is also gratefully acknowledged. The comments by two anonymous reviewers and the Associate Editor were much appreciated and improved this article significantly.

\section{References}

Baldock, T. E., P. Holmes, S. Bunker, and P. van Weert (1998), Cross-shore hydrodynamics within an unsaturated surf zone, Coastal Eng., 34, 173196.

Battjes, J. A., and J. P. F. M. Janssen (1978), Energy loss and set-up due to breaking of random waves, in Proceedings of 16th International Conference on Coastal Engineering, pp. 569-587, Am. Soc. of Civ. Eng., Reston, Va.

Caballeria, M., G. Coco, A. Falqués, and D. A. Huntley (2002), Selforganization mechanisms for the formation of nearshore crescentic and transverse sand bars, J. Fluid Mech., 465, 379-410. 
Calvete, D., N. Dodd, A. Falqués, and S. M. van Leeuwen (2005), Morphological development of rip channels at normal and near normal incidence, J. Geophys. Res., 110, C10006, doi:10.1029/2004JC002803.

Coco, G., T. J. O’Hare, and D. A. Huntley (1999), Beach cusps: A comparison of data and theories for their formation, J. Coastal Res., 15(3), 741749.

Falqués, A., and D. Calvete (2005), Large-scale dynamics of sandy coastlines: Diffusivity and instability, J. Geophys. Res., 110, C03007, doi:10.1029/2004JC002587.

Falqués, A., A. Montoto, and V. Iranzo (1996), Bed-flow instability of the longshore current, Cont. Shelf Res., 15, 1927-1964.

Falqués, A., G. Coco, and D. A. Huntley (2000), A mechanism for the generation of wave-driven rhythmic patterns in the surf zone, J. Geophys. Res., 105(C10), 24,071-24,087.

Holman, R., and A. Bowen (1982), Bars, bumps, and holes: Models for the generation of complex beach topography, J. Geophys. Res., 87, 457-468.

Horikawa, K. (1988), Nearshore Dynamics and Coastal Processes, Univ. of Tokyo Press, Tokyo.

Konicki, K. M., and R. A. Holman (2000), Water-level fluctuations and flow in tidal inlets, Mar. Geol., 169, 69-101.

Mei, C. C. (1989), The Applied Dynamics of Ocean Surface Waves, 740 pp. World Sci., River Edge, N. J.

Niederoda, A. W., and W. F. Tanner (1970), Preliminary study on transverse bars, Mar. Geol., 9, 41-62.

Oltman-Shay, J., and R. T. Guza (1987), Infragravity edge wave observations on two California beaches, J. Phys. Oceanogr., 17, 644-663.

Ribas, F., A. Falqués, and A. Montoto (2003), Nearshore oblique sand bars, J. Geophys. Res., 108(C4), 3119, doi:10.1029/2001JC000985.

Roelvink, J. A. (1993), Dissipation in random wave groups incident on a beach, Coastal Eng., 19, 127-150.
Ruessink, G., I. M. J. Enckevort, K. S. Kingston, and M. A. Davidson (2000), Analysis of observed two- and three-dimensional nearshore bar behaviour, Mar. Geol., 169, 161-183.

Short, A. D. (1999), Handbook of Beach and Shoreface Morphodynamics, John Wiley, Hoboken, N. J.

Soulsby, R. (1997), Dynamics of Marine Sands, 249 pp., Thomas Telford, London.

Thornton, E. B., and R. T. Guza (1983), Transformation of wave height distribution, J. Geophys. Res., 88(10), 5925-5938.

van Enckevort, I. M. J., B. G. Ruessink, G. Coco, K. Suzuki, I. L. Turner, N. G. Plant, and R. A. Holman (2004), Observations of nearshore crescentic sandbars, J. Geophys. Res., 109, C06028, doi:10.1029/ 2003JC002214

van Leeuwen, S. M., N. Dodd, D. Calvete, and A. Falqués (2004), Stochastic processes in beach response predictions, in Coastal Engineering 2004, pp. 2159-2169, Am. Soc. of Civ. Eng., Reston, Va.

Yu, J., and D. N. Slinn (2003), Effects of wave-current interaction on rip currents, J. Geophys. Res., 108(C3), 3088, doi:10.1029/2001JC001105.

D. Calvete and A. Falqués, Departimento de Física Aplicada, Universitat Politécnica de Catalunya, Campus Nord, Modùl B4, E-08034 Barcelona, Spain. (calvete@fa.upc.es; falques@fa.upc.es)

N. Dodd, School of Civil Engineering, University of Nottingham, University Park, Nottingham NG7 2RD, UK. (nick.dodd@nottingham. ac.uk)

S. M. van Leeuwen, CEFAS, Pakefield Road, Lowestoft NR33 0HT, UK. (s.vanleeuwen@cefas.co.uk) 